\title{
INDEX TO VOLUME 123
}

This index provides coverage for both the Initial Reports and Scientific Results portions of Volume 123 of the Proceedings of the Ocean Drilling Program. References to page numbers in the Initial Reports are preceded by "A" with a colon (A:), and those in the Scientific Results (this book), by "B" with a colon (B:).

The index was prepared by Wm. J. Richardson Associates, Inc., under subcontract to the Ocean Drilling Program. The index contains two hierarchies of entries: (1) a main entry, defined as a keyword or concept followed by a reference to the page on which that word or concept appears, and (2) a subentry, defined as a further elaboration on the main entry followed by a page reference.

The index is presented in two parts: (1) a Subject Index and (2) a Taxonomic Index. Both parts cover text figures and tables but not core-description forms ("barrel sheets") or core photographs. Also excluded are bibliographic references, names of individuals, and routine front and back matter.

The Subject Index follows a standard format. Geographic, geologic, and other terms are referenced only if they are subjects of discussion. This index also includes taxonomic entries above the generic level, as well as broad fossil groups such as foraminifers and radiolarians. The notation "ff" following a page listing indicates that reference to a topic continues beyond the last page given but is not sequential. This would be the case where one or more figures or tables follow a principal topic of discussion that makes up a major section. A site chapter in the Initial Reports is considered the principal reference for that site and is indicated on the first line of the site's listing in the index. Such a reference to Site 765 , for example, is given as "Site 765, A:63-267."

The Taxonomic Index is an index relating to significant findings and/or substantive discussions, not of species names per se. This index covers three varieties of information: (1) individual genera and species that have been erected or emended formally, (2) biostratigraphic zones, and (3) fossils depicted in illustrations. A taxonomic entry consisting of both genus and species is listed alphabetically by genus and also by species. Biostratigraphic zones are listed alphabetically by genus; zones with letter prefixes are listed under "zones."

For further information, including available electronic formats, contact the Chief Production Editor, Ocean Drilling Program, 1000 Discovery Drive, College Station, Texas 77845-9547, U.S.A. 
accretionary wedges, sediment subduction, B:167 acoustic impedance

igneous rock, A:330

Site 766, A:311

vs. porosity, B:463

Afar hot spot, Red Sea, B:797

Afar Triangle-Bay of Aden Rift system, volcanism, B: 210

African margin $\mathrm{W}$

palygorskite, B:785

palynology, B:434

Agulhas Current, proto-, Falkland Plateau, B:755

Albian/Aptian boundary, Site 765, A:85

Albian/Cenomanian boundary

Site 765, B:727

Site $766, \mathrm{~B}: 733$

Alexander Island, radiolarians, B:744

alkali, total, vs. silica, B:100

alkali elements, as crustal recycling tracers, B:184

alkalinity

phosphate content and, A:147

Site 765, A:145-146; B:81

Site 766, A:303

alkanes, branched, B:219-221

$n$ - alkanes, B:219

Alpine-Mediterranean Tethys, carbonate sediment, B:751

alteration

basaltic basement, A:189-190, 192-194

igneous rock, A:193, 321-322

stages, A:193-194

stratigraphy, A:193

alteration, high-temperature, Site 765, B:197-198

alteration, low-temperature

geochemistry, B:194-196, 793

patterns of, B:193-194

secondary mineralogy, B:191-193

Site 261, B:23

two-stage evolutionary model, B:191

aluminosilicate, derivation, B:67

aluminum

logging data, B:639

in palygorskite formation, B:70

aluminum oxide

basement basalts, A:187, 189

Cretaceous, B:226

logging data, B:183-184, 188, 641

Site $766, \mathrm{~A}: 323$

vs. zirconium, A:195, 203

ammonium

Site 765, A:147; B:80-81

Site 766, A:303

Ammosphaeroidinidae, Site 766, B:273

andesites, in Sunda ash, B:181

anelastic strain recovery

analytical methods, B:472, 474

Blanton model, B:470-471

elastic properties affecting, B:477

equipment, B:471-473, 489

in low-permeability rocks, B:489

Poisson's ratio, B:471

Site 765, A:206, 209-211

Site 766, A:328-331

stress indicators, B:470

thermal azimuthal anisotropy, B:475-476, 488

thermal stabilization of core samples, B:474475

Warpinski and Teufel model, B:471

Angola Basin, turbidites, B:10

ankerite, vs. calcian dolomite, B:79
Antarctic-Australian continent, glaciation, B:316 apatite

Site 765, A:101; B:180

in smectite, B: 108

Aptian/Albian boundary

Site 765, B:727, 730

Site 766, B:733

total organic carbon, $\mathrm{B}: 218$

aragonite, A:100; B:78, 82-83, 87

Argo Abyssal Plain

calcareous nannofossils, B:41-42, 369-371

depositional history, B:46-48

fan construction, A:106

fracture zones, B:666-668

geological setting, A:4, 65-67; B:151, 660, 662

geomagnetic isochrons, B:550

heat flow, B:515

hemipelagic clay-to-turbidite transition, B:805

igneous geochemistry, B:791

juvenile-mature ocean transition, B:487

magnetic lineations, B:659, 664-668, 792

oceanic crust, $\mathrm{A}: 247$

opening, B:46

paleowater depth, $\mathrm{B}: 812$

radiolarian claystone, $\mathrm{B}: 101$

seafloor spreading, B:102, 810

sediment source, B:168

sediment starvation, B:46

sedimentation history, A:6; B:48, 128, 788

tectonic subsidence, B:685, 687, 697

Tethyan sediment sequences correlated, B:47

volcanic basement, B:804

Argo Basin

carbonate sedimentation, B:752

Cretaceous paleoclimatology, B:753-755

Indo-Australian breakup event, B:316

Jurassic opening, B:753

paleoceanographic events 1-6, B:752-755

proto-circumantarctic current, B:315

radiolarian-rich sediments, B:752

radiolarians, $\mathrm{B}: 315-316$

Argo Ocean, paleogeographic reconstruction, A:3

argon dating, B:558

ash layers

alteration, A:94, 286

bentonites in, A:104

clay mineral composition, A:100

redeposited, B:104, 805

Site 765, A:91, 93

Ashmore Platform, B:689, 693

Astrorhizida

Australian distribution, B:278, 283

Site $766, \mathrm{~B}: 272$

Ataxophragmiidae, absence of, in Australian fauna, B:284

Atlantic Ocean N

benthic foraminifers, B:248-250

tectonic subsidence, B:697

tectono-stratigraphic evolution, B:672

Atterberg limits, B:494-495

augite, basement phenocrysts, A:189

Austral Realm

evidence for, B:747ff

nannofossils, calcareous, B:41

radiolarians, B:313

Australia

benthic foraminifers, B:283-285

palynology, B:423, 433-434

Australia NW palynology, B:423

rain forest, $B: 423-425$

seismicity, B:506

Australia-Antarctica-India, paleogeography, B:533, 535

Australian continental margin, rift-to-drift evolution, B:57

Australian NW margin

Barremian-Aptian convergence zone, B:750

bathymetry, B:524

compressional stress state, B:512-513

continent-arc collision, B:693

Eurasian Plate conversion, B:683

evolution, A:3, 338-339; B:101, 299

geography, A:6; B:802, 740

magnetic lineations, B:661

Mesozoic sedimentation, B:807-812

mineralogy, B:788-789

Oligocene-Miocene unconformity, A:112

paleogeography, B:92, 103

progradation, $\mathrm{B}: 159-160$

seafloor spreading, B:662

sedimentation history, B:811-812

stratigraphic modeling, B:687-693

stratigraphic record, B:682-695

tectonic subsidence, B:684-687, 692-695

turbidite sequences, B:128

upwelling, B:127

volcanism, B:789

Australian Plate. See also Indo-Australian Plate Sunda Arc collision, A:3, 112

Australian Polar Wander Path, A:3; B:552

autobrecciation, Site 765, A:176

Bachus-Naur-Form (BNF), B:609

background pelagic and hemipelagic sedimentation (BPS), Site 765, A:111

Bagginidae, Site 766, B:279

barite

in bentonitic claystone, B:97-98

in radiolarian claystone, $\mathrm{B}: 17-18$

Site 765, A:101

in snarl turbidites, B:121, 125

sulfate in precipitation of, B:786

barium

Site 765, A:160; B:177

vs. calcium carbonate, A:156-157

baroid capillary suction test, Site 765, A:217

Barremian/Aptian boundary

floral/faunal changes, B:740

juvenile-to-mature ocean transition, B:807

magnetic properties, A:138, 140

magnetostratigraphy, B:534

Ocean Anoxic Event (OAE-1), B:234

Site 766, A:288; B:733

Barremian/Hauterivian boundary, Site 766, A:284

Barrow-Dampier Sub-basin

location, B:689

stratigraphic modeling, B:688

tectonic subsidence, B:685, 696

Barrow Group

Australian NW margin, B:683-684

Carnarvon Basin, B:127

Exmouth Plateau, B:127, 578

basal sediments

age, A:269; B:804-805

Argo Abyssal Plain, B:3, 43, 46-48

mineralogy, B:93

Site $765, \mathrm{~B}: 43$

basalt. See also basement, basalt 
geochemistry, B:805

low-temperature alteration, B:793

LREE depletion, B:795

magnesium oxide vs. titanium oxide, A:326

magnetic properties, A:138

mantle melting, B:791, 797

petrophysical logging, A:223-224, 248

physical properties, B:454

Site 766, A:323-324

basalt, holocrystalline, radiometric age dating,

$$
\text { B:558 }
$$

basalt, massive, A:176, 178-179

alteration, B:193

aphyric, A:174-176

basalt, pillow

alteration, B:193

aphyric, A:178-179

brecciated, A:175, 179, 185

magnetic properties, B:551

phyric-aphyric, A:175-176

Site 765, A:173-174

basalt flow

Site 765, A: 176, 178

Site 766, A:316-317

basalt hyaloclastite, Site 765, A:94

basalt intrusion

common parental magma, A:323

fractionated, A:323

geochemistry, A:322-323

Site 766, B:793

trace elements, A:323-324

basalt pebbles

alteration, A:193

in conglomerates, B:203

in Cretaceous sediments, B:68

geochemistry, A:187, 189, 323; B:204-207

occurrence, A:185-186

petrography, A:64, 186-187, 192

Site 765, A:172

source, A:189

velocity, A: 168

basalt/sediment contact. See sediment/basalt con-

basalt sill

clinopyroxene-olivine-plagioclase phyric, A:316

physical properties, A:326

Site 766, A:316-318; B:203

basement

age, B:731, 739

alteration, A:193; B:191, 193-194

coring operations, A:74

geochemistry, A:194-200

geological models, A:272

lithologies, B:557, 793

lithostratigraphy, A:169, 172-181; B:203-204

magnetic vs. biostratigraphic age, B:662

minimum age of formation, B:559

petrography, A:179-189

radiometric age dating, B:191, 557

basement, acoustic, Site 766, B:791

basement, basalt

alteration, A:189-190, 192-193

geochemistry, A:194-201, 203; B:204-206

igneous units 1-24, B:201-203

magnetic properties, A:200-203ff

magnetostsatigraphy, A:201-202

megacrysts and gabbroic fragments, B:201202, 204

mineral chemistry, B:207

N-MORB characteristics, B:204-205, 210

petrography, A:191-192; B:204

physical properties, A:203-206 seismic reflection profiling, A:228

Site 765, A:195-198

stress, A:206-214

basement, oceanic

chemical alteration, A:247-248

oxidative alteration, B:196-197

potassium,argon dating, B:805

Site 765, B:791

basement, volcanic

initiation, $\mathrm{A}: 7-8$

lithologies, A:64; B:792

magnetic anomalies, B:804

seismic reflection profiling, A:4, 66; B:567, 804

Site 765, B:91-92

stress, A:50-52

basement fault blocks, tectonics, A:248, 272

belemnites

biogeography, B:446-448

Boreal/Tethyan affinities, B:446, 751

homeomorphies, B:446

Indian Ocean E, B:443-445

Indonesian affinities, B:446-447

Mediteranean vs. Indo-Pacific provinces, B: 446

preservation, B:445-446

pseudo-apical channel, B:446

in sandy silt/clay, Site 761, B:95

Site 765, A:129; B:9

Site 766, A:297

bentonites

age, B:95

ash turbidite thickness and, B:104

background sediment association, B:97-98

classification, B:91

color and chemical composition, B:94-95

definition, B:89

geographical and structural setting, B:94-95, 97

horizons B1-B6, B:95

in inoceramid sediments, B:9

mineralogy, B:98, 110,785

in Neocomian claystone, A:104

in opal-A and -CT sediments, B:67-68

other sites correlated, A:343

Site 765, A:246

Site 766, A:283; B:806-807

smectite, B:98

source, B:47

tectonic-paleogeographic environments, B: $101-102$

transport distances, B:105

volcanic sources, B:102, 104

beryllium isotopes, Indonesian volcanics, B:186

bioclastic pebbles, displacement, A:289

biomarker geochemistry, B:221-223, 232

biostratigraphy. See zonation, probabilistic; particular microfossil groups; Taxonomic Index

biozonation, calculating optimal, B:760, 762

Blake Bahama Formation, pelagic limestones, B: 751

Blue Mountains, Oregon, Parvicingula sp., B:744

Bolivinidae, Site 766, B:278

Bonaparte Basin, Miocene uplift, B:693

borehole televiewer (BHTV)

operations, A:74

Site 765, A:65, 210, 212-213, 248; B:504, 506 stick-slipping, A:213

temperature measurements, B:516, 519

bottom currents

Indo-Australian straits, B:315

radiolarian assemblage effects, B:312
Bouma sequences

in calcareous sediments, B:20, 28

in radiolarian claystones, $\mathrm{B}: 15,17$

in turbidites, B:118-119, 125, 157, 601, 603

Bowes Canyon, sediment dispersal role, B:128

breccia calcite-cemented, Site 765, A:198

breccia, pillow, A:174, 179

breccia, tectonic, A:193

Brigadier Beds, Australian NW margin, B:683

Brigadier Trend, tectonic subsidence, B:685, 695

British Geological Survey, stress measurement equipment, A:50-51

Broome earthquake

$P$ - axis of focal mechanism, B:506

stress regime, B:511-512

Browse Basin, normal fault reactivation, B:693

Brunhes Chronozone, Site 765, A:132

Brunhes/Matuyama boundary, Site 765, A:132; B:723

Buliminida

Australian distribution, B:281, 284

Site 766, B:276, 278

Cajon Pass, California, wellbore breakouts, B:505

calcareous ooze, transition to chalk, B:784

calcareous sediment

Albian-Miocene, B:18-19

Argo Abyssal Plain, B:46

Cretaceous-Holocene, B:805

depositional environment, B:19, 30-31, 34

Miocene-Pliocene, B:19-20, 33-34

Quaternary, B:20

redeposited, A:76, 80; B:19, 30

Site 765, A:90-91; B:45

sources, B:30, 788

Tithonian-Barremian, B:26-31

Valanginian-Barremian, B:15-17

velocity, B:590-591

vs. noncalcareous, B:751

calcispheres

Argo Abyssal Plain, B:45

in inoceramid sediments, B:8-9, 11

in marl, B:24, 26

in nannofossil claystones, B:24-26

needle formation, B:11

Site 261, B:53

Site 765, A:94, 103, 129; B:53

in smarl turbidites, B:114

in smectite matrix, B:108

in turbidites, B:29

calcite

in alteration veins, A:190, 193

burial diagenesis, $\mathrm{B}: 83$

degree of saturation, B:79, 82

in interbasalt limestones, B:23

precipitation, $\mathrm{B}: 11$

recrystallized, B:87

Site 765, A:98, 100, 149; B:192-193

calcite, low-magnesium, Site 765, B:78

calcite compensation depth (CCD)

Berriasian-Valanginian, A:113

Cretaceous, B:129

cyclic fluctuation, B:30

Jurassic, B:43

sedimentation rate and, B:810-811

Site 765, B:94

Tithonian,B:10-11

water/sediment interface, A:288

calcite needles, Site 765, B:53

calcium

dolomite precipitation and, $\mathrm{B}: 82$

pore-water, B:83

Site 765, A:147 
Site 766, A:303

in smarl turbidites, B: 120

calcium carbonate

in calcareous sediments, B:27-28

Cretaceous sediments, B:226

dilution, B:187

lithologic distribution, A:305

logging data, B:646-649, 656

shear strength and percentage of, B:496

Site 765, A:80, 102-103, 158-159; B:218

stratigraphic variability, A:152

in turbidites, B:493

vs. magnesium oxide, A:159

vs. silica, A: 158

vs. strontium, A: 158

calcium oxide

in basement basalts, A:193, 204

igneous rock, A:325

vs. LOI, A:199

vs. zirconium, A:195

California continental margin $\mathrm{N}$, palynology, B:421

California current, high-latitude radiolarian transport, B:315

Canadian Arctic Islands, continental lithosphere, B:681

Canning Basin

shale, B:181

stress orientation, B:505-506

canyons, submarine

Cretaceous incision, $\mathrm{B}: 20$

sediment transport, B:128, 578

seismic reflection profiling, B:571

Cape Range Fracture Zone, uplift, B:579

carbon, inorganic

Site 765, A:158-159

Site 766, A:305

carbon, organic

accumulation rates, B:227, 231

alkalinity and, A:303

manganese content and, A:303

methanogenic microbial degradation, B:232233

Site 765, A:64, 159-160, 162-164

Site 766, A:308-309

sulfate reduction and, A:147; B:79

carbon, total. See also total organic carbon (TOC)

Site 765, A:158-159

Site $766, A: 305$

carbon dioxide, Site 765, A:161

carbon isotopes, Cretaceous sea level lowstands and, B:754

carbon monoxide, Site 765, A:161, 164

carbon/phosphorus ratios, B:232-233, 235

carbonate

alkalinity, B:80

$\mathrm{CCD}$ and variation in, $\mathrm{B}: 43,47$

in Cretaceous sediments, B:15

cross laminations, A:82

graded sequence, A:89, 106, 279

in inoceramid sediments, B:7

Kimmeridgian-Tithonian deposition, B:751

lithologic units L1-L3, B:24

mineralogy, A:76, 149; B:78-79

predicting petrophysical parameters, B:143144

in radiolarian claystones, B: 15

recrystallization, $\mathrm{B}: 23$

recrystallized, B:74

redeposition, A:40; B:789

Site $765, A: 84 ; B: 47$

in turbidites, A:247; B:82, 114-116, 119-120, $124,158,164$ carbonate, authigenic

in Cretaceous turbidites, B:120, 122, 125

in radiolarian claystones, B:31-32

carbonate, clay-rich, Site 765, A:88

carbonate, diagenetic

formation, B:786

mineralogy, B:77

origin, A:152

in radiolarian claystones, B:14-15, 17

rhombohedral crystals, B:78-79

carbonate, manganese, isotopic composition, B:121

carbonate, neritic, B:140-142, 144-145

pore-system characteristics, B:140-142

carbonate carbon

Site 765, A:162-164

Site 766, A:308-309

carbonate micronodules, radiolarian tests and, B:31

carbonate platform

fragmentation, B:681

Thakkhola, Nepal, vs. NW Australia, B:807, 810

Caribbean Sea, palynology, B:434

Carnarvon Basin

Barrow Delta deposits, B:47

Birdrong Sandstone, B:95

Marssonella Association, B:283

strike-slip faulting, B:693

Carpathians, Polish, benthic foraminifers, B:246, 747

Carpentaria Basin, Ammobaculites Association, B: 283

Casuarinaceae, Site 765, B:422

celadonite

oxygen isotopes, $\mathrm{B}: 197$

radiometric age dating, B:558-559, 805

saponite/iron-oxyhydroxide association, B:197

Site 765, B: 192

celestite, Site 765, B:83

Cenomanian/Albian boundary, Site 765, A:84

Cenomanian/Turonian boundary

calcareous nannofossils, B:383

seismic reflection profiling, A:34

Site $765, \mathrm{~B}: 727$

Site 766, B:733

Cenomanian/Turonian Boundary Event (CTBE), Site 765, A:107, 111

cerium, anomalies, B:179, 18

cesium, Site 765, A:161

Chagos Bank, intraplate stress, B:681, 686-687

chalcedony, in radiolarian-rich turbidites, B:125126,131

chalk

logging data, A:219

Neocomian deposition, A:104-105

physical properties, A:307-308

chalk, calcareous

biogenic components, B:147

Site 765, A:80-8

Site 766, A:283

chalk, calcisphere-nannofossil, Site 761, B:95, 97, 102

chalk, clayey, A:80, 83, 85, 107

chalk, nannofossi

Albian-Cenomanian, A:288

magnetic properties, A:297-298

Site $765, A: 80,83,85$

Site 766, A:280-28

Chenopodiaceae, Australian grassland pattern, B: 423

chert, radiolarian, Site 766, B:755

chert layer, physical properties, A:308, 313 chloride

Cretaceous sediments, B:68

Site 765, A:142-145

Site 766, A:303

chlorite, source, A:150-151

chromium, basement basalts, A:195, 198-199

chromium/aluminum oxide ratio, Site 765 , B:177178,182

Cieszyn Limestones, foraminifers, B:250

Circumantarctic current, B:315-316, 754-755

clasts

Site 766, A:278-279

in smarl turbidites, B:115-116, 127

clay

authigenic, B:147

bentonitic, B:89, 91-94

in Cretaceous turbidites, $\mathrm{B}: 127$

magnesian, B:177-178

silty, A:79

Site 765, A:79, 84, 157: B:89

Site 766, A:280

terrigenous, B:64

zeolitic, A:297-298; B:32-33, 551

clay minerals

assemblages A-E, A:99-101

in bentonites, B:99, 110

detrital, B:64-56

diagenesis, B:66-70, 785-786

elemental composition, B:59, 63-64, 67

with low-temperature alteration, B:191-193

Miocene-Pliocene, B:34

in noncalcareous clay layers, B:20

origin, A:150-152; B:64-70

Site 765, A:84-85, 88-89, 149; B:178

stratification, A:193

claystone

biogeographic implications, A:129-130

bioturbation, A:282-283

black, Site 261, B:32

calcareous, A:91, 246

calcareous vs. noncalcareous, A:91

coloration, A:83, 106

Cretaceous-Paleogene, A:107

greenish-gray, B:529-530

Hauterivian-Aptian, B:805

hematite content, A:136, 138, 140

magnetic properties, A:136, 138, 140, 299; B:551

mineralogy, A:89, 93

physical properties, A:165, 307-308

radiolarian, $\mathrm{B}: 31$

reddish brown, B:529

seismic reflection profiling, A:341-342

Site 261, B:32

Site 765, A:80-8

claystone, bentonitic

age, B:97

clay minerals, B:99

diagenetic alteration, B:105

geochemistry, B:100

Site 765, B:107

Site 766, B:93-94

smectite-rich, B:97-98

type 1-4 B:99-100

volcanic sources, B:100, 105

claystone, calcareous

red/green color boundary, B:9

Site 765, A:86, 90, 94, 104

in smarl turbidites, B:116

Valanginian-Barremian, B:15

claystone, carbonate, Site 261, B:26

claystone, greenish gray, magnetic properties, B:529-530 
claystone, nannofossil depositional environment, B:26

Site 261, B:43-45, 56

Site 765, A:92, 103; B:35

Tithonian, B:24-26

claystone, noncalcareous

depositional environment, B:30-31

Site 261, B:26-31

Site 765, B:15-16

claystone, prodelta, Site 763, B:101

claystone, radiolarian

Barremian-Aptian, B:17-18, 31-32, 45

colors, B:17, 45

depositional environment, B:15, 17-18, 32

graded layers, B:17

Site 261, B:744

Site 765, B:55, 102, 744

Tithonian-Berriasian, B:13-15

claystone, reddish brown, magnetic properties, B:529

claystone, siliciclastic, Site 766, A:282

claystone, silty

calcareous vs. noncalcareous, B:92, 94

with shell fragments, A:102-103

Site 765, A:91-94

Tithonian and older, B:5, 7, 43

claystone, smectite-rich, Site 261, B:30

claystone, zeolitic

Albian-Miocene, B:18-19

Site 261, B:45-46

Site 765, A:83; B:45-46

claystone/basalt contact, Site 765, A:95

clinoptilolite, B:786

in Eocene claystones, A:107

Site 765, A:98, 100

clinopyroxene

basement basalts, B:207

basement phenocrysts, A:179, 183

low-pressure fractionation, B:794

cluster analysis, B:603

color banding

calcareous vs. noncalcareous sediments, B:27

ferrous iron correlation, B:234

lithologic correlation, A:84-85

Site $765, \mathrm{~A}: 89$

Site 766, A:280-281

compaction, inoceramid sediments, B:11

compression index, measured vs. calculated values, B:497

conductive reheating, oceanic crust, B:198

conglomerate, A:38, 41

conglomerate, matrix-supported

Cretaceous/Tertiary boundary, A:107

Miocene, B:19-20, 151-152

physical properties, B:635

Site 765, A:77-79, 81, 83

conglomerate, polymictic, Site 766, A:280

consolidation, B:494-498

continent/ocean boundary (COB)

Argo Abyssal Plain, B:660, 662

Exmouth Plateau, B:575, 801

magnetic anomalies, B:667

Site 766, B:793

copper, vs. calcium carbonate, A:156

Cretaceous quiet zone, Site 765, A:136; B:534, 730

Cretaceous/Tertiary boundary

Australian NW margin, B:684

calcareous nannofossils, B:398

matrix-supported conglomerate, A:107

Site 765, B: 727

Site 766, A:280, 341

crust, continental extensional tectonics, B:801

stress, B:503

crust, oceanic

age of formation, B:575, 577-578

alteration, B:198

conductive reheating, B:191, 198

geochemistry, B:797

permeability, B:521

stress-induced vertical motion, B:673-676

crest, transitional, Site 766, B:577

crustal recycling

Site $765, A: 8$

tracers, B:184-185

Cuvier Abyssal Plain

marine magnetic anomalies, B:659

opening, B:46

sedimentation history, A:6

debris flow, Site 765, A:125, 129, 165, 169

deformation, stress-induced, B:680-682

density

basement basalts, A:204

igneous rock, A:326, 329-330

Site 765, A:164-165, 218, 222; B:456-459, 603-604

Site 766, A:307-309, 315, 334, 336; B:460462

diabase

anelastic strain recovery, A:328-330

intrusion origin, $\mathrm{A}: 331$

physical properties, A:326

radiometric age dating, B:558

Site 765, A:174-175, 190

Site 766, A:270, 318-319

diagenesis. See also silica diagenesis

clay mineralogy, B:784-786

Site 765, B:79-85

smarl turbidites, B:120, 125-126

Dingo Claystone, Australian NW margin, B:683

dinoflagellates

Neogene, B:432-434

Site 765, B:429-432, 434

zonation, B:747

dolomite

aragonite association, B:82

authigenic mineral association, B:79

calcian, B:79

calcium content and, A:147; B:82

carbonate derivation in, B:85

composition, B84-85

degree of saturation, B:79

Miocene, B:79, 786

precipitation, B:77, 82

rhombohedral crystals, B:87-88

Site 765, A:98-100, 103; B:74-75

sulfate concentration and, B:82

Dupal anomaly, Indian Ocean, B:201, 796

Dzong Formation, sedimentation, B:810

earthquakes. See Broome earthquake

earthquakes, normal-fault, B:681, 686-687

Eggerellidae, Site 766, B:273

elastic properties

analytical methods, B:483-485

in-situ confining pressure, B:484-485

moduli, B:474, 488

seismic velocities and, B:490

Ellenburger Formation, recovery efficiency, B:144

Eocene/Oligocene boundary, Site 765, B:725

Eromanga Basin

benthic foraminifers, B:283

Bulldog Shale, B:754

sulfur speciation, B:229 erosion, Site 765, A:83; B:20

erosional truncation, seismic reflection profiling, A:339-340

Europe NW

stress field orientation, B:672

syn-breakup volcanism B:102

eustatic sea level

intraplate stress role, B:673-676

third-order cycles, B:160

turbidite deposition and, B:129, 158-160

Exmouth Plateau

Aptian-Berriasian unconformity, B:95

Barrow Delta deposits, B:47

bathymetry, A:271

breakup, B:805

geologic setting, B:801

heat flow, B:515

mineralogy, B:788

paleowater depth, B:813, 815

Rhaetian-Neocomian nondeposition, B:788

stratigraphic modeling, B:688

stress orientation, B:505

structural setting, B:690-691

submarine canyons, B:128

tectonic history, A:274; B:685, 694

uplift, B:578-579

Valanginian-Aptian hiatus, A:288

volcanic sediments, B:30

Exmouth Plateau Arch, formation, A:67

Exmouth Plateau C

geologic evolution, A:5, 67

sequence stratigraphy, B:805-806

Exmouth Plateau N

Aptian radiolarian chalks, B:127

geologic evolution, A:5-6, 67

Exmouth Plateau $\mathrm{S}$

bathymetry, B:566

volcanic intrusions, B:104

Falkland Plateau

Neocomian sediments, B:752

planktonic foraminifers, B:754

faulting

soft-sediment, A:80

stress regimes and, B:510

ferromanganese crust, in radiolarian claystone, B:13-15, 55

folding, lithospheric

compressional-stress levels, B:675, 680, 688 duration, B:681

foraminifers, aragonitic, Site 765, B:78

foraminifers, benthic

abyssal sediments, A:130

agglutinated, A:104, 121, 139, 246; B:9, 11, $14,722,805$

Ammobaculites Association, B:284

Argo Abyssal Plain, B:42

Australia, B:283-285

Biofacies B assemblage, B:246-247

biogeography, A:139; B:284-285

biostratigraphic summary, A:115-118, 292

calcareous, A:120, 130; B:9

Carpathian assemblages compared, B:246, 250

chronostratigraphy, B:283

deep-water agglutinated, B:239, 244-246, 248-250, 746

depositional environment, B:245-246

isotopic composition, B:84

Marssonella Association, B:283-284, 747

Nepalese assemblages compared, B:246-247

paleodepth, A:294

paleoenvironmental implications, A:129

in radiolarian claystones, $\mathrm{B}: 17$ 
sand fauna, B:245

Site 261, B:247

Site 765, A:120-122; B:135

Site 766, A:294-295, 342; B:271-273, 278 282

size-sorting, A:121

zonation, A:44

foraminifers, planktonic

analytic methods, A:45

Aptian/Albian boundary, A:294

biostratigraphic summary, A:119-118, 292

in calcareous chalk, B:147

chronostratigraphy, B:282-283

Cretaceous, B:721

datum events, A:302

Eocene-Pliocene unconformity, A:342

Mesozoic paleobiogeography, B:755

Neogene, A:42, 116-119, 246, 293; B:719

paleoceanography, B:742, 753-754

Paleocene/Eocene boundary, A:293

paleoenvironmental implications, A:129

Paleogene, A:43, 119-120, 293; B:720

reworking, A:117, 120

Site $765, A: 120$

Site 765, A:293-294; B:731-735

in turbidite sands, B:722

zonation, B:718, 722

fractures

categories, B:477-478, 482-483

crack fillings, B:477

igneous rock, A:319

orientation, A:216, 331

strain recovery and, B:489

stress relationship, A:214, 248, 330-331; B:488-489

zones A-I, B:666-668

francolite, Site 765, B:79-80

gabbroic layer

geochemist, A:323

grain size, A:318-319

Gascoyne Abyssal plain

geologic setting, A:5, 270-272

geomagnetic isochrons, B:550

marine magnetic anomalies, B:659, 662

opening, B:46

seafloor spreading, B:668

sedimentation history, A:6; B:806-807

tectonic subsidence, B:685, 687, 697, 810

geochemical logging

basement, B:644, 650, 652

data reduction, B:638-639

oxide zones 1-3, B:641, 644

Site 765, A:53-54; B:183, 637-644, 646-649

Site 766, A:53-54, 270; B:638, 654, 656

geochemical stratigraphy, lithologic correlation, A:8

geochemistry, inorganic

basement, A:194-200

bentonites, B: 100

bulk composition, B:182-183

Cenozoic vs. Cretaceous sediments, B:188

cotectic control, A:200

igneous rock, A:322-324

lithologic variation, A:152, 322-324; B:169182

major and trace elements, B:170-173

Mid-Indian Ocean Ridge (MIOR) and Red Sea rift lavas compared, B:794-797

sediment source, detrital phase, B:181-182

Site 765, A:64, 142-158, 247; B:174-175

Site 766, A:302-304 vs. mature ocean MORBS and MIORBS, B:794-797

vs. Red Sea basalts, B:794-797

geochemistry, organic

Site 765, A:158-164, 247

Site 766, A:304-306, 343-344

geomagnetic polarity time scale, B:660, 667

geophysical well logs. See logging

George Creek, shale, B:181

Gippsland Basin, sea-level, B:672-673

glaciation

Antarctic-Australian continent, B:316

Australian continent, B:754

glass, volcanic

alteration to smectite, B:67

bubble wall, B: 110

geochemistry, B:205-206

potassium/argon dating, B:805

Site 765, B:202-203

glauconite grains, Site 766, A:286

global optimum zonation, minimum variance scheme, B:604

Globigerinida

Australian distribution, B:282, 284

Site 766, B:277, 280

Globotextulariidae, Site 766, B:273

goethite, Site 765, A:101

Gondwana

burial and subsidence trends, B:810

Cretaceous reconstruction, B:315, 809

du Toit-type reconstruction, B:753

fit and break-up history, B:314-315, 753

Jurassic/Cretaceous reconstruction. A:4; B:748-749

Mesozoic reconstruction, B:807

Tethys Ocean segment, B:299

volcanic evolution, B:89

graded sequence

Aptian, A:105-106

calcareous sediments, A:283; B:19

cross-laminations, A:88

depositional environment, A:108, 111

depositional units, A:87-88

Markov chain analysis, A:106-107

Site 765, A:77-81, 83-84

grain size

calcareous turbidites, B:153, 155-157

gabbroic layers, A:318-319

GRAPE density

Site 765, A:169

Site 766, A:311, 313

graphite, Site 765, A:101

gravity-flow deposits

Cenozoic, B:788

seismic reflection profiling, B:570, 578

Great Artesian Basin, foraminifers, A:294; B:283

halo, alteration

development, B:196-197

distribution, B:196

geochemistry, B:195-197

mineralogy, A:183, 185; B:196-197

in radiolitites, $\mathrm{B}: 15$

with rhodochrosite, A:105

Site 765, A:190, 193, 196-197; B:193, 197

Hamersley Basin, Proterozoic sediments, B:181

Hauterivian/Barremian boundary, Site 766

heat flow A:299; B:735

basalts, B:519

Bullard method, B:517-519

formation fluids and flow-back, B:520

Site $765, A \cdot 241-244 ; B \cdot 515-521$
Site $766, A \cdot 342$

Hedbergellidae, Site 766, B:282

high field-strength elements (HFSE), as crustal recycling tracers, B:185

hopanes, B:222

Hunton Group carbonates, recovery efficiency, B: 144

hyaloclastite

alteration patterns, B:193

breccia, A:184, 193

Site 765, A:173-176

hydrocarbon gases, B:219-221

Site 765, A:161, 164, 170-171

Site 766, A:305-306, 312-313

hydrofracture experiments, A:52-53, 75

hydrogen index

mineral matrix effect, B:217-219

Site $765, \mathrm{~A}: 160$

hydrogen sulfide, Site 766, A:306

hydrothermal convection, B:198

igneous intrusion, Site 766, A:269

igneous rocks

alteration, A:321-322

geochemistry, A:322-324

lithostratigraphy, A:313-319

magnetic properties, A:324ff

petrography, A:319-321

physical properties, A:325-328

stress, A:328-331

illite, Site 765, A:99; B:64

illite/smectite

detrital, B:64

diagenetic, B:66-68

origin, B:785

Site 765, B:58-59, 73-74

Site 766, B:780

structural formulas, B:67

illitization, reaction temperature, B:785

Indian Basin C, unconformities, B:681-682

Indian Ocean

isotopic composition, B:798

juvenile-to-mature evolution, B:788-789

lithosphere, B:673-675

magnesium oxide vs. titanium oxide, A:326

opening, B:102, 345

pelagic sedimentation, B:789

sedimentation rates, $\mathrm{B}: 129$

volcanic basement, A:7-8

Indian Ocean C, stress field, B:680-683

Indian Ocean E, tectonic reorganization, B:668

Indo-Australian Plate

breakup event, B:101, 316

counterclockwise rotation, A:136-138

deformation, B:503

evolution, B:673

intraplate stress, B:509, 513, 674-675, 680, 693

paleolatitude, B:531

Indo-Pacific Province

belemnites, B:446-447

calcareous nannofossils, B:357, 359

inoceramid sediment

bioturbation, B:7, 11

calcisphere-rich layer, B:54

CCD and deposition of, B:10-11

depositional environment, B:9-11, 13

microtextures, B:54

noncalcareous component, B:9

prism textures, $\mathrm{B}: 8$

Site 765, B:43-45, 52

Tithonian, B:7-13

Inoceramidae, Site $765, \mathrm{~B}: 10$ 
inoceramids

bipolar bivalve distribution, B:731

Site 261, B:26

Site 765, B:7-8, 10, 126

interstitial-water chemistry, A:47

Site 765, A:142-149, 247; B:80

Site 766, A:302-306, 344

intraplate deformation seismic reflection profiling, B:680

intraplate stress

Central Indian Ridge/Chagos Bank, B:686687

continental rheology models, B:675, 677-678

deformation changes with, B:681

elastic plate model, B:676-677

in-plane force variation, B:674-675, 679

Indian Ocean NE, B:696

lithological effects, B:684

sea level and, B:671, 673-679

stratigraphic effects of, B:688-689, 692

vertical motion effects of, B:673-676

iron

in Cretaceous clays, B:178

logging data, B:641, 644

Site $765, \mathrm{~B}: 82-83$

iron, ferrous, B:233-234

iron + manganese/aluminum + titanium ratios, Site 765, B:68

iron, pyrite, B:233-234

iron oxide

alteration effects, B:194, 195

aluminum ratio, Cretaceous/Tertiary boundary, B:178-179

basaltic glasses, B:794

basement basalts, A:194

igneous rock, A:325; B:794

magnesium oxide ratio, B:194

Site 765, B:59, 64, 69

Site 766, A:322-323

iron oxyhydroxide, Site 765, B:193, 196

Ivory Coast, palynology, B:434

Jaramillo Chronozone, Site 765, A:126; B:723

Joey Rise

formation, B:104

marine magnetic anomalies, B:666

volcanic pile, B:578

Jurassic/Cretaceous boundary

calcareous nannofossils, B:369-371

Site 261, B:40

juvenile-to-mature ocean transition

Exmouth Plateau, B:805

Gascoyne Abyssal Plain, B:807

Indian Ocean, B:788-789

tectonic subsidence, B:803

Kangaroo Syncline

formation, A:67

stratigraphic modeling, B:702-710

tectonic subsidence, B:685, 694

kaolinite

in calcareous sediments, B:19

detrital, B:64

Site 765, A:99-100; B:608

source, A:98, 150

LAB-TEC 100, vs. pipette procedure, B:153, 155, $158-163$

laminations

B division, B:117, 124, 133

radiolarian-rich, B:118-119

in sandy turbidites, B:122

lanthanum/samarium ratio, basaltic rocks, B:206
Lau Basin, ash turbidites, B:104

lava, geochemistry, A:64

lava/dike transition, albitization of plagioclase, B: 198

Le May Group, pelagic sedimentation, B:752

lead isotopes

basalts, B:794

in Java volcanics, B: 186

vs. Red Sea basalts, B:798

Leg 22

geochemical stratigraphy, B:186

lithostratigraphy, B:186

Leg 27

geochemical stratigraphy, B:186

lithostratigraphy, B:186, 760

mineralogy, B:788-789

Leg 116, sea-level lowstands, B:694-695

Leg 122

mineralogy, B:788-789

objectives, B:801

paleoenvironment, B:808

sea level lowstands, B:694-695

limestone

Argo Abyssal Plain, B:23, 43

magnetic properties, A:298-299

nannofossil, Cretaceous, B:752

linear voltage-displacement transducer (LVDT), B:472

lithosphere

depth-dependent rheology, B:673-675, 684

equivalent elastic thickness, B:687-688

stretching models, B:688-689

lithostates

hand-specimen vs. wireline logs, B:602, 611

transitions, B:604, 616-619

lithostratigraphy. See also under specific sites

basement, A:169, 172-179; B:201-204, 655

Cretaceous, B:527

depositional environment, A:113

Eocene-Pliocene hiatus, A:289

igneous rock, A:173-179, 182-183, 313-319; B:651

Leg 27 compared, B:760

mineralogical correlation, B:57-59, 62, 565566, 568-570, 780, 783-784, 787

Pliocene hiatuses, A:113

sediment source, B:578-579

seismic correlation, A:341-342; B:568-570

Site 765, A:76-94, 109-110; B:96, 493

Site 766, A:269, 276-286, 288; B:493, 655

Unit I, A:76-79, 96, 112, 277-279; B:603, 605

Unit II, A:80-82, 111-112, 219, 279-281; B: $143-144,572,574,603,605-607$

Unit III, A:82-83, 96, 98, 107-111, 219-220, 281-285, 287-288; B:570-572, 578, 806-807

Unit IV, Site 765, A:83-88, 96, 105-106, 219220

Unit V, Site 765, A:88-90, 105, 219-220

Unit VI, Site 765, A:90-91, 104-105

Unit VII, Site 765, A:91-94, 102-104

Lituolida

Australian distribution, B:279, 283

Site 766, B:272-273

Locker Shale Formation, Australian NW margin, B:683

logging. See also geochemical logging; petrophysical logging

basalt, A:222-223

cased-hole section, A:222-223

lithoporosity, A:217

lithostratigraphic correlation, A:219-220

$\log$ quality, A:221-222 seismic correlation, A:217, 228-234

Site 765, A:9, 53-54, 72-76, 215-221, 248, 253-267

Site 766, A:53-54, 331-334, 346-352

Londonderry Arch, location, B:689

loss on ignition (LOI), basement basalts, A:199

Lower Campanian Event (LCE), Indian Ocean, A:111

lysocline, Quaternary fluctuation, B:30

microfossils, Cretaceous, B:747

Madeira Abyssal Plain, Quaternary turbidites, B: 129

magma

geochemistry, B:796-797

magnesium oxide-rich, A:7

magnesium

in palygorskite sediment, B:785-786

replacement reaction, $\mathrm{B}: 82$

Site $765, \mathrm{~A}: 147$; B:82-83

Site 766, A:303, 308

vs. calcium carbonate, A:156, 159

magnesium oxide

aluminum oxide ratio, $\mathrm{B}: 177$

basement basalts, A:194

igneous rock, A:325

silica ratio, A:159

titanium dioxide ratio, A:326; B:69

Site 765, A:159, 160, 326; B:69, 177

vs. LOI, A:199

magnesium oxide/aluminum oxide ratio, Site 765 , B: 177

magnesium oxide/silica ratio, Site 765, A:159

magnesium oxide/titanium dioxide ratio, Site 765 , A:326; B:69

magnetic polarity time scale

Cretaceous, B:523-525

polarity chron nomenclature, B:524

magnetic properties, A:46

basement, A:200ff

calcareous turbidites, B:152, 157, 164

characteristic direction, B:525-526, 529, 542548

Cretaceous, B:529-530

Cretaceous-Paleogene, A:133, 136-139

demagnetization, B:528-529, 550-551

igneous rock, A:324-325ff

linear decay to the origin, B:529-530

mean destructive field (MDF), A:200

mean inclinations, A:139-140, 202-203, 207209

Neogene, A:130-133

NRM intensity, A:132-133, 200, 299, 324

Site 765, A:14-16, 130-140; B:529

Site 766, A:14-16, 297-301; B:529-530

susceptibility units, A:131-133, 136, 297 299, 325

thermal demagnetization behavior, A:200; B:529

viscous remanent magnetization (VRM), A:324

magnetite, A:299; B:207

magnetostratigraphy, A:42-43

analytical methods, B:525-527

Aptian polarity chronozones, B:534-538

Barremian-Aptian hiatus, B:735

basalt basement, A:201-202

biostratigraphic correlation, B:525, 624

Cenozoic, B:725, 727

Chronozone Cl-C9, A:132-133; B:723, 725

Chronozone C12, A:133; B:725

Chronozone C13, A:136

Chronozone C21-C28, A:136 
Chronozone C32-C33, A:136

Chronozone M0, B:534, 730

Chronozone M1, B:534, 538, 730, 733

Chronozone M2, B:538

Chronozone M3, B:538, 730, 733

Chrorrozorres M3-M11, B:538-539

Chronozone M5-M18, B:537

Chronozone M8, B:730

Chronozone M10, B:735

Cretaceous, A:299-301; B:534-539, 719, 727731,733-735

Cretaceous-Paleogene, A:133-139

Mesozoic, A:46

Neogene, A:132-133; B:719

Paleogene, B:719

Site 765, A:7, 136-138; B:96

Malita Graben, B:689

manganese

carbonate precipitation and, A:156-157; B:82

in Cretaceous clays, B:178

Site 765, A:147; B:83

Site 766, A:303

in smarl turbidites, B:120-121

manganese carbonate

mineralogy, B:17

in radiolarian claystone, B:18

manganese nodules

in claystone, $\mathrm{A}: 105$

in inoceramid sediments, B:9, 11, 54

manganese oxide

aluminum ratio, Cretaceous/Tertiary boundary, B:178-179

in calcareous sediments, B:19

Site 765, A:93-94, 160

manganese oxide/aluminum ratio, Cretaceous/Tertiary boundary, B:178-179

manganese oxide crust, Site 765, A:93-94

manganite, Site 765, A:99, 101, 103

mantle melting

continental rift margins, B:791

geochemical effects, B:797, 805

partial, B:209-210

Marin Headlands Terrane, radiolarians, B:744

marine magnetic anomalies

across fracture zone, B:666, 668

correlation, tectonic history, B:667-668

Hawaiian pattern, B:523

M-sequence, B:523-524, 664

Mesozoic, B:659

observed vs. calculated, B:66-

Site 765, A:4, 74, 15, 66, 138,2 46-247; B:46, 527, 665-667, 730-731, 804

Site 766, A:8, 299, 301; B:527, 577, 806

Markov chain analysis

cyclic sequences, A:95-97

graded carbonate sequence, A:106-107

self-transitions, B:608

Site 765, B:602

turbidites, A:111-112; B:144

marl, calcisphere-nannofossil

bentonitic, B:102

Site 761, B:95, 97

marl, calcite needle-rich, Site 261, B:26 marl, nannofossil, Site 261, B:23-25, 43

mercury porosimetry, B:138-139

metabentonites, clay mineralogy, B:68

metal, calcium carbonate and, A:152, 156

methane/ethane ratio, Site 766, A:306

methylsteranes, Site 766, B:221-222

Mexico, Gulf of, palynology, B:434

mica, Site 765, A:100, 103; B:64

micrite, Site 765, A:102-103

microcracks, B:482, 484-485 microfossils

abundances, B:745

assemblages 1-9, B:767

bipolar distribution, B:755

with lithologic changes, B:752

probabilistic zonation, B:759, 765-772

zones, B:764-767

Mid-Indian Ocean Ridge basalt (MIORB)

Atlantic and Pacific mid-oceanic ridge sites compared, B:791-792, 796

Red Sea basalts compared, B:791

Site 765, A:194

mid-ocean ridge basalt (MORB)

isotopic composition, B:796

melting, B:791

oceanic comparisons, A:12

Site 765, A:187

Miliolida, Australian distribution, B:280, 283

mineralogy, A:47

Argo Abyssal Plain, B:786, 788

associations I-IV, B:780, 783

associations I-V, B:780, 783-784

associations I-VII, B:57-59

Australian NW margin, B:788-789

diagenetic effects, B:784-786

lithologic correlation, B:57-59, 62, 787-789

regional correlation, B:788-789

secondary, A:183, 185; B:191-193

Site 261, B:780, 782-784

Site 765, A:47, 149-152, 247; B:60-61, 603

Site 766, B:780-781

zeolitic clays, B:32-33

Miocene/Pliocene boundary

magnetic properties, A:133

stratigraphic hiatus, B:723

Monterey Formation, B:67, 70, 84

Muderong Shale, B:95, 97

Mungaroo Formation, B:683

Myrtaceae, Site 765, B:422

nannofossils, calcareous

Albian/Aptian boundary, A:124

analytic methods, A:44-45

Argo Abyssal Plain, B:42, 370-371

Austral Realm, B:41

Barremian-Albiann homogenization, B:755

biogeography, A:130; B:34, 41-42, 48, 351, 355-356, 370-371, 740-741, 743, 755

biostratigraphy, A:115-118, 302; B:35-41, 382-383, 392-403

Boreal affinities, B:41, 350-351, 359

in calcareous chalk, B:147

Campanian/Maestrichtian boundary, A:123

Campanian-Santonian hiatus, A:124; B:727

Cenomanian/Albian boundary, A:124

Cenomanian/Turonian boundary, A:291; B:383

Cenozoic, A:123-125, 289-291; B:731

in claystones, A:104; B:25

Cretaceous, A:291-293; B:344-355, 384-387, 392-398, 721, 731-735

Cretaceous/Tertiary boundary, B:398

Cretaceous unconformities, B:392, 397, 401

in debris flows, A: 125,129

distribution, B:346-349, 371-377

Eocene hiatus, A:289; B:394, 401

etching, A:103; B:36

Gault, B:356-357

in inoceramid sediments, $\mathrm{B}: 11$

Jurassic/Cretaceous boundary, B:40, 369-370

Mesozoic, A:124-125

Miocene unconformity, A:123

Neogene, A:42; B:719
Oligocene hiatus, B:395

Oligocene/Miocene boundary, B:395

paleoceanography, B:742, 752-754

Paleocene/Eocene boundary, B:399

paleoenvironmental implications, A:129, 289; B:41-42, 351, 355-356

Paleogene, A:43; B:388-391, 399-404, 720

Paleogene/Eocene boundary, B:402-403

Pliocene hiatus, A:122; B:400

provincialism, A:130; B:740

recrystallized, B:135

regional correlation, $\mathrm{B}: 351$

reworking, A:122

Site 765, B:8-9

temperate assemblage, A:289

Tethyan affinities, B:41-42, 351, 357, 359, $741,743,755-756$

Tithonian evolutionary radiation event, B:40

in turbidites, B:28-29, 114-115

Valanginian/Hauterivian boundary, A:342

zonation, A:41; B:357, 359-360, 717-718

Nassellarians, faunal provincialism, A:130

natural gamma-ray spectrometry, A:218, 222,334

neodymium isotopes, B:794, 798

neutron porosity (NPHI), A:219, 222, 336; B:6CB604

New Zealand, taxa, B:744

nickel

in basement basalts, A:195, 198-199

in turbidites, A:161

Ninetyeast Ridge, stress-induced deformation, B: 680

niobium, Site 765, A:199-200

niobium/yttrium ratio, vs. zirconium/titanium dioxide, B:101

nitrogen gas, Site 765, A:161

Nodosariida

Australian distribution, B:280, 283-284

Site $766, \mathrm{~B}: 273,278$

nontronite, B:197

North Sea, siltstone, B:47

Northwest Australian Shelf. See Australian NW margin

Oates Canyon, B:104, 128

Olduvai Chronozone, Site 765, A:126

Oligocene/Miocene boundary, Site 765, B:395, 725

olivine, basement phenocrysts, A:179

Oman, radiolarites and claystones, B:751

ooze, bioclastic, B:140

ooze, calcareous

with clay, A:76

physical properties, A:165, 307

Site 766, A:280

ooze, nannofossil

Albian-Cenomanian, A:288

calcium carbonate content, A:305

clayey, A:78, 81

Site 765, A:78

Site 766, A:277-280, 282

ooze, siliceous

Argo Abyssal Plain, B:46, 48

Pliocene-Quaternary, B:34

Quaternary, B:20

opal-CT

lepispheres, B:74

Site $765, \mathrm{~A}: 150$

transformation from opal-A, A:148; B:784 786

opaques, Site 765, B:5

organic carbon/total sulfur ratio (C/S), B:227$232,234-235$ 
organic matter

maturity, B:222

origin, B:788

terrestrial, B:219

orthopyroxene, basement basalts, B:207

overconsolidation ratio (OCR), B:494, 496, 498

oxides, major-element

basement basalts, A:201

sediment, A:154

oxygen index, Site 765, A:160; B:218

oxygen isotopes, alteration products, B:197

$P$ - wave velocity, B:487-489

Site 765, A:169

vs. in-situ, B:633-634

Pacific Ocean, dinoflagellates, B:433

Paleocene/Eocene boundary, Site 766, B:399, 402-403, 731

paleocurrents, Site 766, B:580-581

paleoenvironment, structural evolution and, B:808 paleolatitude, B:527

Aptian-Albian, B:755

Australian Polar Wander Path compared, B:531-533, 552-553

basement basalts vs. sediments, B:553

Jurassic, B:753

mean inclinations, B:530-531, 552

Mesozoic, A:46

Site 765, A:139-140

paleowater depths, Australian NW margin, B:810813

palygorskite

mineral associations, B:69, 79, 785

ooze-to-chalk transformation, B:784

origin, B:70, 785

Site 765, A:98, 100; B:74-75

source, A:151-152

in zeolitic clays, B:33

palynology

abundance, B:422

Antarctic assemblages, B:747

Aptian-Albian source area, A:129

Aptian/Barremian boundary, A:125

Argo Abyssal Plain, B:42

Australia, B:423, 433-434

biostratigraphy, A:118, 292; B:407-410

Cenozoic, B:422-425

Cretaceous, A:125-126; B:232, 721

distribution, B:413-415

grassland species, B:423

in marine sediments, $\mathrm{B}: 421$

Neogene, A:125, 246; B:432-434

paleoenvironmental implications, A:129

Persian Gulf, B:434

Peru, B:434

in radiolarian claystones, $\mathrm{B}: 17$

reworking, A:126

Site 261, B:27

Site 765, A:118-119; B:424-426

Site 766, A:297

tropical, B:432-434

turbidity-current-deposited, B:421

zonation, A:44; B:722

pantanellids, Site 765, A:130

Papuan Basin

Favusella, B:284

planktonic foraminifers. B:747

parent/daughter ratios, as crustal recycling tracers, B: 185

parvicingulid spp., Site 765, B:323, 337

passive continental margins, magmatism, A:7

Patellinidae, Site 766, B:278

permeability oceanic crust, A:56-57

Site 765, A:244

Persian Gulf, palynology, B:434

Perth Abyssal Plain, marine magnetic anomalies, B:659, 662

Peru, palynology, B:434

petrofacies, units I-IV, B:141-142

petrography

basement, A:179-189

groundmass, A:183, 185,321

igneous rock, A:319-321

phenocrysts, A:179, 183, 319-320

xenocrysts and xenoliths. A: 185

petrophysical logging

basalt, A: 248

lithostratigraphic correlation, A:336

Site 765, A:64; B:604

Site 766, A:270, 344

units 1-8, Site 765, A:223-224

units 1-11, Site 766, A:336-337

$\mathrm{pH}, \mathrm{A}: 145-146,303$

phosphate

barite correlation, B:100, 102

diagenesis, B:79

in pelagic clays, B:180-181

rare earth element control by, B:179

Site 765, A:147, 160; B:81

Site 766, A:303

phosphorus, vs. calcium carbonate, A:156-157

photoelectric factor (PEF)

measured vs. calculated, B:637

Site 765, A:218-219

physical properties

basement basalts, A:203-206, 210-211

data discrepancies, B:454

igneous rock, A:325-328, 344

seismic correlation, A:168-169

Site 765, A:164, 168-169ff; B:494-496

Site 766, A:270, 307-313ff, 344; B:494-496

units A-E, A:165, 169, 248, 307-309, 313, 326,344

plagioclase

albitization, B:197-198

alteration products, B:193

basement basalts, A:179, 182; B:195, 207

low-pressure fractionation, B:794

plagioclase-clinopyoxene pairs, anorthite-Mg\# rerelationship, B:207, 211

plagioclase megacrysts

basement basalts, B:204

glass inclusions, B:207, 211, 213

plate tectonics

Argo Basin, B:315-316

Cretaceous models, B:753-755

Pliocene/Pleistocene boundary

seismic reflection profiling, B:589

Site $765, \mathrm{~B}: 723$

Poaceae, Australian grassland pattern, B:423

porcellanite, Site 761, B:97

pore-system characteristics

analytical methods, B:138

of lithified carbonates, B:137

petrophysical analysis, B:139

turbidites, B:140, 142-143

porosity

acoustic impedance predictions, B:463-465

basement basalts, A:204

calcareous chalk, B:149

density relationship, B:455

igneous rock, A:326, 330

physical property vs. logged values, A:222

recrystallized wackestone, B:149

Site 765, A:113, 164-165; B:456-459
Site 766, A:307-309; B:460-462

thermal conductivity relationship, B:464

porosity-velocity transforms

acoustic formation factor, B:463

compaction correction factor, B:461

linear regression equations, B:454-455, 459, 461-464

post-Archean Australian Shale (PAAS), B:181183

potassium

Site 765, A:148-149; B:639, 641

Site 766, A:304; B:639

potassium/argon dating

basement, B:805

celadonite, B:559

rhyolitic rock, B:792, 803

volcanic sediments, A:7

potassium content logs, B:603-604

potassium oxide

alteration effects, B:194

basement basalts, A:193-194, 199; B:197

logging data, B:184, 188, 641

Site 765, A:161; B:178

vs. chromium, B:181-182

precision depth recorder (PDR), A:14, 21-22

preconsolidation stress, MGC vs. Casagrande methods, B:496-497, 501

pristane/phytane ratio, $\mathrm{B}: 221$

prodelta deposits, sea level lowstands and, B:805

progradational wedge, Site 766, A:286, 343

proto-Agulhas Current, Falkland Plateau, B:755

proto-circumantarctic current system, Argo Basin, B: 746,754

pyrite

formation, B:227-228, 786

Site $765, \mathrm{~A}: 150 ; \mathrm{B}: 80,87,230-231$

Site 766, A:284, 299; B:230-231

pyrite sulfur, vs. organic carbon, B:231

pyritization, degree of (DOP), total organic carbon and, B:233-234

pyroxene, basaltic rocks, B:208

quartz

detrital, B:87

in inoceramid sediments, B:9

Site 765, A:99, 101-102

radiolarians

abundance, B:302-303, 306-307

analytical methods, A:45; B:300-301, 303

Aptian-Albian, B:755

Argo Abyssal Plain, B:42-43, 744-746

Argo Basin, B:315-316

Austral Realm, B:313

Barremian-Albian homogenization, B:755

Barremian/Aptian boundary, A:295; B:311

biogeography, A:129-130; B:313, 315, 743 744,755

biostratigraphy, A:115, 118, 292; B:303-305, 307-311

bipolar distribution, B:743

in calcareous chalk, B:147

carbonate replacement, B:27-30, 125-126

circumantarctic species, B:315

clay faunas, A:130; B:14, 17, 31, 311-312, 744

Cretaceous, B:721

current-deposited, B:300

Jurassic paleolatitude, B:743

lithology, B:311

Neogene, A:42; B:719

Northwest Australian Shelf, B:32

paleoceanography, B:47, 742, 752, 754 
paleoecology, B:311-312

Paleogene, A:43; B:720

Parvicingula vs. Ristola type, B:313, 744

with pelagic carbonate influx, B:752

preservation, B:302-303, 306-307

proto-Antarctic Ocean migration, B:315

provincialism, B:313

Quaternary, A:126, 246, 295

recrystallized, B:55, 132, 134-135

reworking, A: 126

sand faunas, A:130; B:311-313, 315, 744

sea level lowstands, B:316

Site 765, A:126-129; B:9, 303-305

Site 766, A:295-297, 342; B:733, 735

Tethyan affinities, B:299-300, 311, 313, 759 756

thanatocenoses, B:743

in turbidites, B:115, 246

upwelling effects, B:127, 313, 315

zonation, A:44; B:722

radiolarite

depositional environment, B:15, 746

permeability, B:31

sedimentary structures, B:312

Site 765, A:90ff, 105, 130; B:14, 94, 300, 311

in turbidites, B:111, 114

rain forest, Australia NW, B:423-425

Rankin Trend

stratigraphic modeling, B:702-710

tectonic subsidence, B:685, 695

rare earth elements

basaltic rocks, B:206, 797

Cenozoic vs. Cretaceous samples, B:179-180

chondrite-normalized, B:180

Cretaceous sediments, B:178-181

as crustal recycling tracers, B:184-185

fractional crystallization, B:794, 796

shale-normalized, B:180

recovery efficiency (RE), vs. pore-system characteristics, B: 144

Red Sea, B:794-797

resistivity, A:219, 222, 336

rhodochrosite

authigenic formation, B:121

Cretaceous, A:105, 246

elemental composition, B:84-85

ghosts, B:308

manganese and, A:147; B:83-84

Site 765, A:89-90, 98-100, 103, 127, 152; B:82, 752-753

in smarl turbidites, B:120-121

rhodochrosite, calcian, Site 765, B:79

rhythmic sedimentation, Atlantic Ocean basins, A:7

rift-flank tilting, Wombat Plateau, B:803

rifting history

Australian NW margin, B:792-793

Cretaceous, B:210, 212

Site 766, A:5

Robertiniida, Australian distribution, B:281, 284

Rock-Eval pyrolysis

Cretaceous sediments, B:232

Gascoyne Abyssal Plain, B:219

Site 765, A:160-161, 166-168; B:216-218

Site 766, A:305, 310-311; B:216

Roo Rise

formation, B:104

fracture zones, B:667

volcanic pile, $\mathrm{B}: 578$

Rotaliida

Australian distribution, B:281, 284

Site 766, B:276, 278-280

rubidium alteration effects, $\mathrm{B}: 194$

basement basalts, A:193, 199; B:197

rubidium/samarium ratio, carbonate content and, B: 185

$S$ - wave velocity, B:487-489

salinity

Site 765, A:142-145, 147

Site $766, \mathrm{~A}: 303$

samarium

in pelagic clays, $\mathrm{B}: 180$

vs. aluminum oxide, B:182

samarium/neodymium ratio, carbonate content and, B: 185

samarium/ytterbium ratio, Cenozoic vs. Cretaceous samples, B:179, 183

sand, ferruginous, transgressive, B:95

sand, glauconitic, paleoceanographic correlation, B:752

sand, radiolarite, bottom current winnowing, B:312

sand, volcanic, shallow-water source, B:578

sandstone, Site 766, A:283-284

sandstone, calcareous, Valanginian-Barremian, B: 15

sandstone, clayey, Site 765, A:94

sandstone, glauconitic

physical properties, A:308-309

Site 766, A:284, 299; B:101

sandstone, polymictic, B:141-144, 150

sandstone, silty, Site 766, A:285-286; B:94

sandstone, volcaniclastic, Site 766, B:101

saponite

celadonite/iron oxyhydroxide association, B: 197

Site 765, B:59, 70, 74

Scott Plateau

Argo Basin sediment contribution, B:47

pelagic marl, B:127

potassium/argon dating, B:792

stress orientation, B:505

submarine canyons, B:128

volcanic sediments, A:7; B:30

volcanism, B:104

sea level

Australian NW margin, A:3

glacio-eustatic role, B:316, 671-673

tectonic subsidence and, B:693-695

Tertiary sedimentation rate and, A:113

third-order cycles, B:671-672, 693-694

seafloor spreading

Argo Abyssal Plain, B:667-669

Australia and Greater India, B:810

Australian NW margin, B:682-683

Valanginian-Hauterivian, B:806

seafloor weathering, Site 765, B:196

seawater-rock interaction, water/rock ratios, B:191

sediment/basalt contact

Site 765, A:93, 102; B:173, 184, 201

Site 766, A:284

Tithonian, B:5

sediment gravity flow

Neogene, A:111-112

tectonic influence, B: 158

sediment starvation

CCD position and, B:810-811

Miocene turbidites, B:612

Site 765, B:803-804

sediment subduction

beryllium isotopes and, B:167

estimating fluxes, B:167

Sunda Trench, B:185-187 sedimentary sequence, Argo Abyssal Plain, B:46 Site 765, B:43-46

Unit L1, B:5, 7, 23-24, 43

Unit L2, B:7-13, 24-26, 43-45

Unit L3, B:13-17, 26-31, 45

Unit L4, B:17-18, 31-32, 45, 48

Unit U1, B:18-19, 32-33, 45-46

Unit U2, B:19-20, 33-34

Unit U3, B:20, 34, 46

sedimentation rate

Argo Abyssal Plain, B:43, 48, 129, 667-668, 810

Australian NW margin, B:789

Cretaceous, B:26

decompacted, A:113-114, 289

effect of vertical crest motion on, B:673-675, 688

inoceramid sediments, B:11

magnesium diffusion and, B:786

Site $261, \mathrm{~B}: 12$

Site 765, A:113, 140-143; B:12, 18, 129, 215 601,725

Site 766, A:270, 301-302; B:215

Valanginian-Hauterivian vs. Aptian turbidites, B:127

zeolitic clays, B:33

sedimentology. See also Maskov chain analysis smear slide analysis, A:40, 101-102

segregation vesicles, Site 765, A:183; B:204, 213

seismic reflection profiling

A-reflector, B:684

B-reflector, B:684

Blue reflector, A:338

C-reflector, B:684

Green reflector, A:338-339

intrabasement, B:567, 577

Red reflector, A:338

Site 261, A:16

Site 765, A:16, 68

Site 766, A:16-17; B:807

seismic stratigraphy

Aptian-Cenomanian sequence, B:572, 579

Barremian-Aptian unconformity, B:572

Barremian sequence, B:571-572, 578

Cenomanian-Turonian unconformity, B:574

Cenozoic sequence, B:574-575, 581

Cretaceous sequence, B:574, 580

erosional truncation, A:226

F-unconformity, B:683

lithostratigraphic correlation, A:248, 341-342, 344; B:565-566

Paleocene unconformity, B:574

regional unconformity, A:231

sequences 1-5, Site 766, A:339-342

sequences 1-7, Site 765, A:225-235; B:154

sequences 3-5, Site 765, B:152

sequence 4-6 hiatus, Site 261, B:788

sequence boundaries, B:565, 589, 597

Site 765, A:248

tectonic-stratigraphic correlation, A:338

Valanginian-Hauterivian sequence, B:567, 570-571, 577-579

seismic velocity, B:483-485

air- vs. water-gun sources, B:584-585

elastic properties and, B:490

lithologic correlation, B:634-635

slant stacking, B:626, 628-629

t- $p$ analyses, B:625

variation with pressure, B:485-487

$X$ - $T$ to t- $p$ mapping, B:626, 628

sepiolite

mineral associations, B:69, 79

source, A:151-152 
sequence analysis. See also syntax analysis chaotic system, B:608

core-description data, B:609-610, 612, 614615

entropy function, B:604

ergodicity, B:604, 608

exogenous subdivisions, B:604

predictability in, B:604

Site 765, B:602, 604-609

wireline-log data, B:610-611, 616-620

shale, black

physical properties, A:326

Turonian/Cenomanian boundary, A:107

shear strength

Site 765, A:169, 177; B:494, 496

Site 766, A:313-314, B:496

shell debris, Site 765, A:94, 102-103

siderite, Site 765, B:79

silica

ash layers, B:103

in bentonites, B: 110

in clay, A: 158

diagenesis, A:149-150, 152; B:70, 786

dilution, B:169, 177, 187

during sepiolite-palygorskite formation, B:69

logging data, B:641

in palygorskite sediment, B:785-786

in radiolarian tests, $\mathrm{B}: 786$

Site 765, A:148, 161

Site 766, A:304

stratigraphic variability, A:152

vs. calcium carbonate, A:158

vs. magnesium oxide, A:159

vs. strontium, A: 158

vs. total alkali, B:100

vs. zirconium, A:195

silica/aluminum oxide ratio, Site 765 , B:177

silica diagenesis, A:149-150, 152; B:786

radiolarian tests and, B:70

temperature, B:70

siliciclastic sediment, Cretaceous, A:104-105

silt, terrigenous, B:64

siltstone

black, A:299, 301, 313

clayey, Site 765, A:91, 93-94, 103; B:92, 94

glauconitic, A:308-309; B:101, 752

sandy, Site 766, A:284, 286; B:94

volcaniclastic, Site 766, B:101

Site 100 , basal sediments, A:12

Site 105

basal sediments, A:12

benthic foraminifers, $\mathrm{A}: 44$

biostratigraphic events, B:770, 776

microfossils, B:763

Site 167, carbonate sediment, B:752

Site 211, clastic turbidites, B:186-187

Site 212, calcareous turbidites, B:186-187

Site 213, siliceous oozes, B:186-187

Site 249, Neocomian sediments, B:752

Site 257

Ticinella, B:747

Ticinella multiloculata, B:284

Site 258

Crucibiscutum salebrosum, B:356

planktonic foraminifers, B:271, 285, 750, 754

Ticinella, B:747

Site 259

basalts, A:201

foraminifers, B:271

lithostratigraphy, B:787

mineralogy, B:788

palygorskite, B:785

titanium/zirconium ratio, B:210
Site 260

basalts, A:200-201

basement, B:661

foraminifers, B:271

fracture zones, A:8

geomagnetic isochrons, A:8

lithostratigraphy, B:787

mid-ocean ridge depths, A:6, 67

mineralogy, B:788

palygorskite, B:785

planktonic foraminifers, A:294

tholeiitic basalt, A:5

titanium/zirconium ratio, B:210

Site 261

basal sediments, A:7, 12, 64, 67; B:3, 43, 46, 48,789

basalt, A:195, 200-201, 203, 205

basement, B:661-662

bathymetry, A:14, 18-20

benthic foraminifers, A:45; B:239, 247, 722

bio-magnetostratigraphy, A:6-7

biostratigraphic events, B:771, 777

calcareous nannofossils, B:37-41, 369-371, 373-377, 730

calcareous turbidites, B:186-187

carbonate banding, A:7

clay mineral diagenesis, B:785

Crucibiscutum salebrosum, B:356

depositional history, B:46-48

fracture zones, A:8

geochemistry, MIOR basalts, A:199-200

geomagnetic isochrons, $\mathrm{A}: 8$

lithostratigraphy, A:70; B:44, 527, 787

location, A:69; B:4

magnetic properties, B:529

magnetostratigraphy, B:538-539

marine magnetic anomalies, A:66-67

mid-ocean ridge depths, A:67

mineralogy, B:780, 783-786, 788

oceanic crust, B:803

paleolatitude, B:530-533, 549-553

paleowater depth, B:810, 812

palygorskite, B:785

radiolarian sands, B:746

radiolarians, B:308, 311-312

reddish brown claystone, A:136

sedimentary sequence, B:20-34

smectite, B:47

titanium/zirconium ratio, $\mathrm{B}: 210$

Tubodiscus verenae, B:764

uplift, A:248

Site 262, dolomite, calcian, B:79

Site 263

basement, B:661

foraminifers, $\mathrm{B}: 271$

fracture zones, $\mathrm{A}: 8$

geomagnetic isochrons, A:8

lithostratigraphy, B:787

mineralogy, B:788

Site 327

Nannoconus, B;755

Ticinella, B:755

Site 330, basal sediments, A:12

Site 335, temperature, B:519

Site 367

basal sediments, A:12

biostratigraphic events, B:771, 777

Site 370

biostratigraphic events, B:770, 776

Hedbergella delrioensis, B:765

Lenticulina nodosa, B:764

Site 386

radiolarite sand, A:105; B:312 rhodochrosite, A:105

Site 387

basal sediments, A:12

biostratigraphic events, B:771, 777

microfossils, B:763

Site 391

benthic foraminifers, B:246

biostratigraphic events, $\mathrm{B}: 770$

calcareous nannofossils, B:35

microfossils, B:763

Site 395

oceanic crust, A:56; B:503, 521

temperature, B:519

wellbore breakouts, B:509

Site 396

alteration halos, B:196-197

temperature, B:519

Site 398, biostratigraphic events, B:771, 776

Site 416

basal sediments, A:12

basement, B:791

benthic foraminifers, $\mathrm{B}: 246$

biostratigraphic events, B:770, 776

calcareous nannofossils, B:735

Site 417

alteration halos, B:196

basement, B:791

lithostratigraphy, A:172

Site 418

alteration halos, B:196

lithostratigraphy, A:172

Site 454 , temperature, B:519

Site 504

basalts, B:511

crustal reheating, B:198

lithostratigraphy, A:172

oceanic crust, A:56; B:503, 521

temperature, B:519

wellbore breakouts, B:509

Site 511

Corollithion silvaradion, B:356

Crucibiscutum salebrosum, B:356

Nannoconus, B:741, 755

Ticinella, B:747, 755

Site 534

basal sediments, A:12

benthic foraminifers, A:44; B:246, 248-250, 747

biostratigraphy, B:759, 770, 775

calcareous nannofossils, B:35

Dorothia praehauteriviana, B:764

geomagnetic polarity study, B:763

microfossils, B:763

Site 535

basal sediments, A:12

biostratigraphic events, B:770, 776

Site 540, basal sediments, A:12

Site 543

benthic foraminifers, B:246

Hormosina ovulum gigantea Zone, B:727

Site 544, basal sediments, A:12

Site 545, basal sediments, A:12

Site 546, basal sediments, A:12

Site 547, basal sediments, A:12

Site 548, basal sediments, A:12

Site 603

basal sediments, A:12

benthic foraminifers, A:44; B:246, 722, 746

biostratigraphy, B:759, 770, 775

calcareous nannofossils, B:359

geomagnetic polarity study, B:763

microfossils, B:763, 764

Site 638 , calcareous nannofossils, B:359 
Site 639 , basal sediments, A:12

Site 641 , benthic foraminifers, A:44; B:246, 722 , 746

Site 692, Cruciellipsis cuvillieri, B:741

Site 693

Crucibiscutum salebrosum, B:356

radiolarians, B:313, 744, 746

Ticinella, B:747

Site 735, oceanic crust, A:56; B:521

Site 759, location, A:11; B:90

Site 760

basal sediments, A:12

location, A:11; B:90

Site 761

ash layers, A:104

basal sediments, A:4, 12

belemnites, B:443-448

bentonites, A:104; B:47, 95, 97

juvenile ocean sequence, B:89

lithostratigraphy, B:787

location, A:11; B:90

Neocomian chalk, B:94

Rhaetian lagoonal facies, B:802-803

Triassic neritic carbonates, B:141

Site 762

basal sediments, A:12

belemnites, B:443-448

location, A:11; B:90, 564

Valanginian-Hauterivian hiatus, B:579

Site 763

basal sediments, A:12

belemnites, B:443-448

bentonites, B:97

Cenomanian-Turonian boundary event, B:805

location, A:11; B:90, 564

Paleocene-Eocene unconformity, B:805-806

Valanginian-Hauterivian hiatus, B:579

Site 764

basal sediments, A:12

location, A:11; B:90

Rhaetian reef complex, B:801-802

Site 765, A:63-267

age dating, B:191

basal sediment, A:12; B:3, 43, 46, 48, 789

basalt, B:204-207

basement, A:4; B:201-203, 661-662

bathymetry, A:14, 18-20

benthic foraminifers, B:242-250, 722

biostratigraphic events, B:771, 777

calcareous nannofossils, B:35-37, 344-351, 355-356, 369-373, 382-395, 400403, 717-718

chronostratigraphy, B:717, 722-731

depositional history, B:46-48

dinoflagellates, B:429-434

geochemical reference section, B:183-185, 188,644

geochemistry, inorganic, A:64, 142-158, 247; B:167-175, 792-796

geochemistry, organic, A:158-164, 247; B:217-221, 226-235

geological setting, B:168-169, 191, 803-804

geological time scale, A:6-7

lithostratigraphy, B:44, 113, 527, 602-604, 787

location, A:3, 9; B:4, 112, 152, 168, 226, 504 , 516,792

magnetic lineations, B:659-669

mineralogy, B:57-64, 786, 788

navigation, $\mathrm{A}: 13-14$

objectives, A:3-4; B:225, 523, 584

paleolatitude, B:530-533, 549-553

paleowater depth, B:810, 812 palynology, B:407-410, 421-425, 432-434, 722

planktonic foraminifers, B:718, 722

radiolarians, B:302-305, 307-313, 315, 722

radiometric age dating, B:557-559

regional geology, A:4

sedimentary sequence, B:5-20

sedimentation history, A:102-113, 149

sequence analysis, B:604-611

vertical seismic profiling, B:589-600

wellbore breakouts, B:505-513

Site 766, A:269-352

basal sediments, A:12; B:789

basalt, B:204-207

basement, B:203-204, 661

bathymetry, A:14, 20; B:567

belemnites, B:443-448

benthic foraminifers, B:722

biostratigraphic events, B:771, 777

calcareous nannofossils, B:350-355, 392-404, 717-718

chronostratigraphy, B:717, 731-735

depositional history, B:580

foraminifers, B:271-285

geochemistry, inorganic, A:302-304; B:793796

geochemistry, organic, A:304-306, 343-344; B:217-221, 226-235

geological time scale, A:6-7

juvenile ocean sequence, B:89

lithostratigraphy, B:527, 529,787

location, A:3, 9; B:90, 226, 563-564, 793

navigation, $\mathrm{A}: 13-14$

objectives, A:34; B:225, 523,563

paleolatitude, B:530-533, 549-553

paleowater depth, B:810, 813, 815

palynology, B:722

planktonic foraminifers, B:285, 722

radiolarians, B:306-307, 310-311, 722

radiometric age dating, $\mathrm{B}: 557-559$

regional bathymetry, B:563

regional geology, A:5

sedimentology, A:286-289, 343

seismic stratigraphy, B:565-575

smectite

in bentonitic claystones, B:99

Cretaceous, A:99

derivation, B:47

dioctahedric, B:108

honeycomb texture, B:108-109

illitization, B:68, 785, 789

Site 765, B:192

source, A:99-100, 151

in volcaniclastic sandstone, B:108

volcanogenic origin, B:17

smectite/kaolinite boundary, Site 765, B:608

sodium dioxide

basement basalts, A:204

vs. zirconium, A:195

sodium-montmorillonite

Site 765, B:99-100

sonic $\log , \mathrm{B}: 603-604$

correlation, sonobuoy and VSP data, B:634635

Site 765, A:218, 222; B:633-634

Site 766, A:334

VSP correlation, B:589-591, 600

sonobuoy seismic recording

correlation, VSP and sonic log, B:634-635

exact normal moveout, B:628-631

operations, A:75-76

Site 765, A:17, 20, 23, 25

Site 766, A:20, 23-24 $\tau-p$ analyses, $\mathrm{B}: 626$

$\mathrm{X}-\mathrm{T}$ to $\tau-p$ mapping, B:626-628

spinel, B:207-208

spreading rate, magma fractionation and, B:210, 212

strain

principal orientation, B:472, 474-475, 478481

temperature correlation, $\mathrm{B}: 476$

STRATCOR program, B:760-762

stratigraphic modeling, Northwest Australian Shelf, B:687-693, 697, 711-713

stress

anelastic strain recovery, A:50-51, 206, 209211

basement basalts, A:206-214

circumferential, B:511-512

Cloetingh and Wortel model, B:506, 509

effective vertical, B:499-500

hydrofrac tests, A:52-53

igneous rock, A:328-331

in-situ directions, A:51-52, 210, 213-214

indicators, B:470

maximum horizontal compressive, B:505, 508, 512

oceanic crust, A:50-52

principal horizontal magnitudes, B:470-471

regime constraints, $B: 509-512$

regional indicators, B:505-506

Richardson model, B:506, 509

strontium

aragonite content and, B:78

basement basalts, A:187, 189, 195

pore-water, B:83

Site 765, A:147-148, 160

Site 766, A:303-304, 323

vs. calcium carbonate, A:156, 158

strontium isotopes, basalts, B:207, 212, 794, 209

submarine fan deposition

models, B:128, 143-144

periodic oscillatory behavior, B:612

Site 765, B:601

Site 766, A:269

subsidence. See tectonic subsidence

sulfate

in barite precipitation, B:786

dolomite precipitation and, B:82

Site 765, A:146-147; B:81, 217

Site 766, A:303

sulfate reduction, organic carbon content and, B:79

Sunda-Banda Arc, Pliocene collision, Australian margin, B:151, 158

Sunda Shelf, continental lithosphere, B:681

Sunda Trench, crustal fluxes, B:167

Surat Eromanga, benthic foraminifers, B:283

Swan Canyon

incision, B:48

pelagic sediment, A:106

as sediment source, B:111, 151

syntax analysis, B:602

environmental model, B:609

parsed sequence, B:611

pattern recognition, B:608-609

Site 765, B:620-623

tectonic subsidence

Australian NW margin, A:3

basin formation effects, B:697

Indian Ocean, B:693

lithological effects, B:684-687

magnetic lineation correlation, B:667-668

Northwest Australian Shelf, B:693-695, 701 
tectonics, basement blocks, A:248, 272

tectonics, extensional, Permian, B:801

temperature

logging data, A:241, 245

Site $765, \mathrm{~B}: 515-518$

thermal gradients, B:520-521

temperature logging tool (TLT), A:241, 342; B:515-517

terrigenous-pelagic sediment transition, Site 766, A:288, 343

Tethys Himalaya, syn-breakup volcanism, B:102

Tethys Ocean, radiolarians, B:299

Tethys Realm. See also nannofossils, calcareous; radiolarians

NW Australian margin biostratigraphy correlated, B:41-42, 739-755

Tethys Sea, tectonic history, A:3

Thakkhola, Nepal

benthic foraminifers, B:246-247

Mesozoic sedimentation, B:807-812

paleoenvironment, B:808

polar wander path, B:807

sedimentation rate, $\mathrm{B}: 816$

thermal conductivity

basement basalts, A:205

igneous rock, A:327, 329, 331

lithologic correlation, B:519

porosity predictions from, B:464

Site 765, A:169, 177-178, 210; B:466-467

Site 766, A:313, 315; B:464, 468

thermal equilibrium gradient, A:56

thermal subsidence, Australian NW margin, B:789

thorium, B:639

thorium/aluminum oxide, $\mathrm{B}: 182$

thorium $\log , \mathrm{B}: 604$

thorium/uranium ratio

carbonate content and, B:185

Site 765, A:219

titanium dioxide/aluminum oxide ratio, Site 765 , B: 182

titanium dioxide/zirconium ratio, altered basalts, B:194

titanium oxide

iron oxide correlation, A:195

Site 765, A:180; B:796

Site 766, A:322-323, 325

vs. calcium carbonate, A:156

vs. carbonate, A:152

vs. major oxides, A:194, 203, 326

titanium oxide/aluminum oxide ratio, clinopyroxene, B: 210

titanium oxide/zirconium ratio

basaltic rocks, B:205

Site 765, A:203

titanium oxide-iron oxide/magnesium oxide correlation

fractional crystallization model, B:210

partial melting trend, B:209-210

Site 765, B:207, 209

titanium/zirconium ratio, basaltic rocks, B:210

Toolebuc Formation, Rock-Eval pyrolysis, B:218

total organic carbon (TOC)

Barremian-Albian Ocean Anoxic Event, B: 234

Site 765, A:159-160, 247; B:217-219, 227, 229-231

Site 766, A:311, 343-344; B:227, 229-231 transition-probability analysis, B:602

classification by information content, B:604

Troodos Ophiolite, basement, B:559

Tryal Rocks, tectonic subsidence, B:692-693 turbidites
Bouma sequences, A:111; B:118-119, 125 $157,601,603$

calcium carbonate content and, A:159

carbonate mineralogy, B:78

derivation, A:104

ergodic mixed carbonate-siliciclastic cycle. B:609-611

fan models, A:112

geochemical variation, A:157-158

metal content, A:157-158

microfossils, B:722

Miocene sedimentation rate, $\mathrm{B}: 601$

organic geochemistry, A:247; B:217-218

physical properties, A:165, 168-169

redeposition mechanism, $\mathrm{B}: 806$

sediment sources, A:106; B:94, 601

small-scale cycles, B:611-612

stacked, B:611

Tithonian-Barremian, B:27

turbidites, ash, thickness and volume, B:104-105

turbidites, calcareous

$\mathrm{CCD}$ and fluctuations in, B:48

classification, A:40-41

Cretaceous-Pleistocene, A:40-41

eustatic sea level and, B:47

magnetic properties, A:132-133; B:722

Miocene, B:157-161, 725

redeposition mechanism, B:30

seismic reflection profiling, A:231

Site 261, B:29

Site $765, \mathrm{~A}: 111$

source, B:18, 30-31, 34, 45, 47, 788, 805

Valanginian-Barremian, B:17

turbidites, calciclastic

analytical methods, B:152-156

effect of sea level on, B:158-160

lithology, B:151-152

logging data, B:152

seismic correlation, B:152

sequence boundaries, B:151-152

turbidites, carbonate

petrofacies analysis, B:141-144

pore-system characteristics, B:140, 142-143

recovery efficiency, B:144

Site 765, A:246

turbidites, greenish gray, depositional environment, B:527, 529

turbidites, mud, in radiolarian claystones, B:18, 32 turbidites, pelagic, sea level and influx of, B:754

turbidites, siliciclastic, source, B:805

turbidites, smarl

Aptian, B:123-126

biogenic components, $\mathrm{B}: 135$

coarse-grained, B:115-122, 124-126, 134

compositional layering, B:117-118, 124

Cretaceous sediment sources, B:126-128

depositional models, B:115, 118-120, 124125,128

diagenesis, B:120-121, 125-126

eustatic lowstand correlation, B:128

facies patterns, B: 126

fine-grained, B:114-115, 123-124

radiolarian-rich intervals, $\mathrm{B}: 131-132$

sandy microtextures, B:119-121

thickness, B:126

Valanginian-Hauterivian, B:111, 114-122

turbidity current deposits, Site 765, A:106; B:610

Turonian/Cenomanian boundary

laminated shale, A:107

Site 766, A:280

Turrilinidae, Site 766, B:278

United States SE, palygorskite, B:785 upwelling, radiolarian assemblage effects, B:313, 315

uranium, B:639

uranium $\log$, B:604

uranium/potassium ratio, logging data, A:219

Valanginian/Hauterivian boundary, Site 766 , A:301; B:735

vanadium/titanium ratio, intrusives, A:326

veins, clay mineralogy, A:322-323

veins, alteration, Site 765, A:173, 190, 192-193

velocity. See also seismic velocity

acoustic impedance predictions, B:463-464

basement basalts, A:205

igneous rock, A:326-327, 329-330

mud cake formation, B:591

Site 765, A:168-169; B:456-459

Site 766, A:309, 311, 314; B:460-462

velocity, interval

from semblance in $X-T, \mathrm{~B}: 630,632$

Site 765, B:588, 591

from VSP data, B:632-634

weighted-average, B:633, 635

velocity anisotropy, B:486-487

comparison, anelastic strain recovery, B:487488

vertical seismic profiling (VSP)

acquisition system, A:237-238

attenuation and dispersion, B:591

basement and sub-basement reflections, B:592

correlation, sonic log and lab velocities, B:589-591, 597, 600

correlation, sonobuoy and sonic log data, B:634-635

data processing, B:587-589

deconvolved seismogram, B:589, 598-599

operations, A:74-75

receivers, B:585-586, 593

recording, B:586-587

scientific applications, B:583

seismic sources, B:584-585

Site 765, A:65, 235-241ff, 248

summed traces, B:586

waveform processing, B:588-590, 592, 594596

zero-offset A:235-237

vesicle fillings

Site $765, \mathrm{~A}: 193$

Site 766, A:318, 321-322

vesicles, zeolite-filled, basement basalts, A:199

vesicularity, basement basalts, A:183

volcanic detritus, in inoceramid sediments, B:11

volcanic intrusion, magnetic properties, A:301

volcanic pebbles

Site 766, A:278-279

transportation, A:289

volcanic pile, Site 766, B:577-578

volcanic rocks

geochemistry, B:793-796

rhyolitic, potassium/argon dating, B:803

volcanism

Australian NW margin, A:7; B:789, 792-793

Cretaceous, mineralogic evidence, B:67

post-breakup, B:89, 102, 104

Wallaby Plateau

igneous geochemistry, B:792

origin, B:104

water content

basement basalts, A:204

igneous rock, A:326, 330

lithostratigraphic correlation, B:494-495

Site 765, A:164-165; B:454-459, 494 
Site 766, A:307-309; B:460-462, 494

Weddell Sea

Neocomian sediments, B:752

planktonic foraminifers, B:754

radiolarians, B:746

wellbore breakouts

intervals 1-3, B:505

principles, B:503-504, 509-511

Site 765, B:512

Wharton Basin

manganese oxide-rich clay, B:187 stress-induced deformation, B:680

Whim Creek, shale, B:181

Windalia Radiolarite, B:744, 746, 755

Winning Group, mineralogy, B:788

wireline $\log$

$\mathrm{N}$-value, B:608

seismic correlation, B:805

Site 765, B:602, 616-623

vs. core-description lithostates, B:611

Wombat Plateau

alkaline rhyolites, B:792

bentonites, B:104

Callovian-Oxfordian tectonic event, B:810

juvenile ocean sediments, B:101 nannofossil-calcisphere chalk, B:45

Neocomian chalk, B:45, 127

paleowater depth, B:811

post-Rhaetian evolution, B:802-803

Rhaetian carbonate sequence, B:801

seafloor spreading, B:803

sedimentation history, B:801-803

seismic reflection profiling, B:803

tectonic subsidence, B:685, 696

volcanic basement, A:7

wood emulsion equation, B:461-462

World Stress Map Project, B:671-672

X-ray diffraction, A:98-101

$\mathrm{X}$-ray fluorescence

intrusives, A:324

Site $765, A: 150,156$

xenoliths, Site 765, A:185, 189

yttrium, Site 765, A:199-200

zeolite

formation, B:786

Site 765, A:103; B:19, 192

Site 766, A:280, 288 zinc, Site 765, A:161, 199

zirconium

in basement basalts, A:180, 195

lavas, B:796

Site 765, A:199-200

titanium oxide correlation, A:195

in turbidites, A:161

vs. major-element oxides, A:203-204

vs. trace elements, A:205

zirconium/titanium dioxide ratio, vs. niobium/yttrium, B:101

zirconium/titanium ratio, intrusives, A:326

zirconium/yttrium ratio, Site 765, A:200, 326

zonation, probabilistic

computation, B:764-767

correlation, Leg 27, B:759-772

Final Composite Standard Sequence (FCSS), B:764, 767, 769

RASC Optimum Sequence, B:765-766

smoothing factor, B:765

zones, fossil. See in Taxonomic Index under zones (for letter prefixes) and alphabetically (for genetic-specific designations)

\section{TAXONOMIC INDEX}

abrupta, Trochammina, Site 765, A:122; B:250, 256, 261-262

Acacia pollen, Site 765, A:125

Acaeniotyle diaphorogona B:317, 332

Acaeniotyle sp., B:317, 332

Acaeniotyle umbilicata, B:317

Site 765, B:332

Acanthocircus, B:317

Acanthocircus trizonalis, B:317

Acanthocircus trizonalis dicranacanthos, B:317

Acarinina mckannai, Site 766, A:293

Acarinina spinuloinflata/Acarinina bullbrooki lineage, Site 765, A:120

Achomosphaera neptuni, Site 765, B:418

Achomosphaera sp., Site 765, B:438

achylosus, Corollithion, Site 766, B:367

acostaensis, Neogloboquadrina, coiling change, A:119

aculeata, Ramulina, Site 765, B:264

aculeus, Ceratolithoides, Site 765, A:124

acutiangulata, Tristix

Site 416, A:295

Site 766, A:295

Adnatosphaeridium tutulosum, Site 765, B:418

alazanensis, Cibicidoides, Site 765, A:121

albiensis, Hayesites

Site 765, A:124

Site 766, A:293

Aldorfia deflandrei, Site 765, B:420

Aldorfia sp., Site 765, B:417

Alievium helenae, B:317-318

Site 766, A:295 allomorphinoides, Quadrimorphina, Site 766, B:290, 294

altispira, Dentoglobigerina, Site 765, A:117

Amaurolithus delicatus, Site 766, A:289

Amaurolithus delicatus Subzone

Leg 63, A:289

Site 766, A:289; B:731

Ammobaculites Association

Australia, B:283

biogeography, B:283

global distribution, B:284

Ammobaculites sp., Site 765, B:245, 251, 261

Ammobaculoides carpathicus, Site 765, B:252, 261

Ammobaculoides sp., Site 765, B:250

Ammodiscoides sp., Site 765, B:252, 258

Ammodiscus cretaceus, Site 765, B:252, 258

Ammodiscus sp., Site 765, B:252, 258

Ammodiscus tenuissimus, Site 765, B:252, 258

Ammolagena clavata, Site 765, B:14, 245, 258

Ammosphaeroidina sp., Site 766, B:292

Amphipyndax, B:318

Amphipyndax mediocrix, B:318

Amphirhopalum ypsilon Zone, Site 765, A:126; B:723

amphitreptera, Podocapsa, B:324

Site 765, A:128, 141-142; B:308-309, 339

Andamookia, Eromanga Basin, B:284

andersoni, Gavelinella, Site 766, B:279, 290, 295

angulare, Anthocyrtidium, reworking, A:126

angulisuturalis, Globigerina, Site 765, A:119

Angulobracchia media, B:318

Site $765, \mathrm{~B}: 332$
Angulobracchia sp., B:318

Site 765, B:331-332

angustus, Rhagodiscus

Site 765, B:359, 368

Site 766, B:351, 359, 366

Ansulosphaera helvetica Zone, Site 765, A:125

Anthocyrtidium angulare, Site 765, A:126

Anthocyrtidium angulare Zone

Site 765, A:126

Site 766, A:295

Anthocyrtis angulare Zone, Site 765, B:723

apertura, Globigerina, Site 765, A:117

apiarium, Archaeodictyomitra, B:318

Site 765, B:332

apicopaucicum, Druggidium, B:765

appenninica Rotalipora, Site 766, B:282, 291, 297

Aprobolocysta galeata, Site 765, B:420

aptica, Hedbergella, Site 766, B:281, 291, 296

aptiense, Globigerinelloides, Site 766, B:280, 290, 295-296

aptiensis, Globorotalites, Site 766, B:290, 294

Aragonia semireticulata, Site 766, A:294

Archaeodictyomitra apiarium, B:318

Site 765, B:332

Archaeodictyomitra brouweri, B:312, 318

Site 765, B:330, 332

Archaeodictyomitra brouweri alpha, B:318

Site 765, B:332

Archaeodictyomitra brouweri gamma, B:318

Archaeodictyomitra brouweri typica, B:318

Site $765, \mathrm{~B}: 332$

Archaeodictyomitra slitera, Site 765, B:730 
Archaeodictyomitra sp., B:318-319

Site 765, B:332

Arenobulimina-Voloshinoides Complex, Site 766, B:273

Arenoturispirillina jeletzkyi, Site 765, B:252, 258

areolata, Systematophora, Site 765, B:416

argoensis, Cyclagelosphaera, n. sp., B:371

Site 261, B:40, 378, 741

Site 765, B:741

argoi, Blysmatodinium, gen. et sp. nov., Site 765 , B: 434,440

Arkhangelskiella cymbiformis Zone, Site 766, A:291; B:732

arnace, Exochosphaeridium, Site 765, B:418 asarotum, Cribroperidinium, Site 765, B:417-418

Aschemocella sp., Site 765, B:252, 259

Ascodinium sp., Site 765, B:407, 418

asper, Rhagodiscus, B:743

Aspidolithus parcus constrictus

Site 765, A:123; B:727

Site 766, A:291; B:732

Aspidolithus parcus parcus, Site 766, A:291

Aspidolithus parcus Zone

Site 765, A:124; B:392, 727

Site 766, A:291; B:732

Astacolus scitula, Site 766, B:278, 288, 293

Astacolus sp., Site 766, B:288, 293

asterigerum, Oligosphaeridium, Site 765, B:416

asymmetricus, Discoaster, Site 766, A:289

attadalense, Scriniodinium, Site 765, B:417

Aulacoteuthis, morphological trends, B:446

australis, Charltonina, Site 766, B:290, 294

australis, Muderongia, Site 765, B:416

Avellodinium sp., Site 765, B:418

avita, Didymocyrtis, reworking, A:126

barbadoensis, Pseudohastigerina, Site 765, A:119

barbui, Holocryptocanium, Site 765, A:128, 130, 142; B:43, 310, 730

barbui barbui, Holocryptocanium, B:312, 321

Site 765, B:336

barnesae, Watznaueria, B:350

Site 261, B:37

barnetti, Cibicidoides, Site 765, A:121

barremiana, Gavelinella, Site 766, A:295

barremiana, Lenticulina, Site 766, B:764

Bathysiphon sp., Site 765, B:252, 257

Batiacasphaera imperfecta, Site 765, B:419-420

Batiacasphaera micropapillata, Site 765, B:440

Batiacasphaera sp., Site 765, B:419

Batioladinium micropodum, Site 765, B:418

Batioladinium reticulatum, Site 765, B:418

Batioladinium reticulatum Zone

Site 761, B:450

Site $763, \mathrm{~B}: 450$

Site 765, B:42, 350, 408, 410, 730

Timor Sea, B:410

Belemnopsis, B:444

Indo-Pacific Province, B:446

regional differentiation, B:446

Site 761, B:97

Belemnopsis gladiatoris, Ethiopian-Antarctic Subprovince, B:446

Belemnopsis jonkeri, B:444-445, 450

Belemnopsis madagascariensis-B. castarensis Group, Ethiopian-Antarctic Subprovince, B:446

Belemnopsis moluccana, B:445

Site 763, B:450

Belemnopsis moluccana-B. stolleyi Group, IndoPacific Province, B:446

Belodinium dysculum, Site 765, B:420 bentonensis, Globigerinelloides, Site 766, B:280, 290, 296

Berthelina intermedia, Site 766, B:279, 290, 294

bettenstaedtii, Eprolithus, n. sp., B:360

Site 766, B:366, 368

biforaminis, Flabellites, Site 766, B:359, 364,368

Bigenerina sp., Site 765, B:252, 263

bigotii, Stephanolithion, Site 261, A:7; B:37, 40, 42, 351, 378

Bilingulogavelinella, Australian distribution, B:284

binaiensis, Globoquadrina, Site 765, A:119

Biretisporites eneabbaensis Zone, Site 766, A:297

Blysmatodinium argoi gen. et sp. nov., Site 765 , B:434, 440

Blysmatodinium gen. nov., Site 765, B:434

Bolivinoides parvissimus, Site 765, B:727

bradyana, Planularia, Site 766, B:289

britannica, Watznaueria

Site 261, B:37

Site 765, A:125

Site 766, B:365

Broinsonia signata, Site 766, B:364

Broomea simplex, Site 765, B:418

brouweri, Archaeodictyomitra, B:312, 318 Site 765, B:330, 332

brouweri alpha, Archaeodictyomitra, B:318 Site 765, B:332

brouweri gamma, Archaeodictyomitra, B:318

brouweri typica, Archaeodictyomitra, B:318

Site 765, B:332

brustolensis, Vitorfus, B:326

Buccinosphaera invaginata Zone, Site 765, A:126; B:723

Buchia, bipolar bivalve distribution, B:751

buddencanyonensis, Lingulina, Site 766, B:288

Bulbobaculites inconstans, Site 765, B:249, 252253,260

bulloideus, Spiniferites, Site 765, B:429-430

buxtorfi, Planomalina

Site 765, A:120

Site 766, A:294; B:280-281, 290, 296

cachensis, Crucella, B:319

Site $765, \mathrm{~B}: 333$

Calcicalathina oblongata Zone, Site 761, B:97

Calcidiscus macintyrei Zone, Site 766, A:289; B:731

Calculites obscurus Zone, Site 765, A:124; B:392, 727

Calculites ovalis Zone

Site 765, A:124; B:392, 727

Site 766, B:397

Campylosphaera eodela, Site 766, B:731

Campylosphaera eodela Subzone, Site 766, A:289; B:399

Candeina nitida, Site 765, A:119

Canninginopsis intermedia, Site 765, A:125; B:407, 419

caracolla, Epistomina, Site 765, B:264

carniolensis, Lithraphidites

Site 261, B:351

Site $765, \mathrm{~B}: 350,730$

carpathicus, Ammobaculoides, Site 765, B:252, 261

Carpodinium granulatum, Site 765, B:417

Cassiculosphaeridia magna, Site 765, B:416

Cassiculosphaeridia sp., Site 765, B:420

Cassigerinella chipolensis, Site 765, A:119

castanea, Trinchodinium, Site 765, B:418

Catinaster calyculus Subzone, Site 765, A:123

Catinaster coalitus Zone, Site 765, A:123; B:723

Cecrops septemporatus, B:319 cenomana, Schackoina, Site 765, B:290, 296

Cenosphaera spp., B:319

Site 765, B:333

Ceratolithoides aculeus, Site 765, A:124

Ceratolithoides aculeus Zone, Site 765, A:124; B:392, 727

cerviculum, Dingodinium, Site 765, B:419

cetia, Sethocapsa, B:325

Site 765, A:128, 141-142; B:43, 341, 730

Charltonina, Australian distribution, B:284

Charltonina australis, Site 766, B:290, 294

charoides, Glomospira

Site 261, B:56

Site $765, \mathrm{~B}: 253$

chenodes, Mirifusus, B:321

Site $765, \mathrm{~B}: 336$

Chiasmolithus bidens Subzone, Site 766, A:289290

Chiasmolithus danicus Zone

Site 765, A:123

Site 766, B:399, 731

Chiasmalithus danicus-Ellipsolithus macellus zones

Site 765, B:393

Site 766, B:399

chiastius, Microstaurus, Site 765, B:344-345

Chiastozygus litterarius, B:767

Site 766, B:364, 368

Chiastozygus litterarius Zone

Site 765, A:124; B:383, 727

Site 766, A:293

chipolensis, Cassigerinella, Site 765, A:119

choanophorum, Melitasphaeridium, Site 765 , B: 431,440

Chondrites, Site 261, B:27

cibaoensis, Globorotalia, Site 765, A:119

Cibicidoides alazanensis, Site 765, A:121

Cibicidoides barnetti, Site 765, A:121

Cibicidoides dutemplei, Site 765, A:121

Cibicidoides havanensis, Site 765, A:121

cisownicensis, Pseudoreophax, Site 765, B:255, 263

Citharina sp., Site 766, B:288, 293

clavata, Ammolagena, Site 765, B:14, 245, 258

Clavihedbergella simplicissima, Site 766, B:282, 290, 296

Clavihedbergella sp., Site 766, B:282, 290, 296

Clinapertina inflata, Site 766, A:294

Coccolithus miopelagicus Subzone, Site 765, A:123

colligata, Epelidosphaeridia, Site 765, B:419

colligata, Speetonia

Site 261, B:741

Site 765, B:350, 367, 730, 741, 743

Site 766, B:741, 743

collignoni, Marginulopsis

Site 416, A:295

Site 766, A:295

Coliosphaera tuberosa Zone, Site 765, A:126; B:723

columbaria, Eucyrtis, B:320

Site 765, A:127; B:335

Site 766, A:295

columbaria, Eusyringium, Site 766, B:311

columnata, Prediscosphaera

Site 765, B:359

Site 766, A:293; B:359

Cometodinium whitei, Site 765, B:420

conica, Thanarla, Site 765, A:129-130

conicus, Cretarhabdus, Site 766, B:365

Conorboides hofkeri, Site 766, A:295

Conusphaera mexicana, Site 261, B:370

Conusphaera mexicana minor 
Site 261, B:37, 40, 741

Site $765, \mathrm{~B}: 741$

cooperi, Zeugrhabdotus, n. sp., B:372-373

Site 261, B:29, 379, 741

Site $765, \mathrm{~B}: 741$

cornuta, Scampanella, Site 766, B:367

Corollithion achylosus, Site 766, B:367

Corollithion kennedyi, Site 765, A:124; B:727

Corollithion silvaradion, Site 511, B:356

corona, Repmania, Site 766, B:287, 292

coronoadtventis, Grantarhabdus, Site 766, B:365

Coryphostoma sp., Site 766, B:278, 289, 293

crassa, Hormosina, Site 765, B:254, 259

crassaformis, Globorotalia, Site 765, A:118

crenulatus, Rotelapillus, Site 766, B:366

cretaceus, Ammodiscus, Site 765, B:252, 258

Cretarhabdus conicus, Site 766, B:365

Cretarhabdus spp., Site 261, B:351

cretosa, Heterantyx, Site 766, B:273, 287, 292

cretosa, Spiroplectammina, Site 766, B:273

Cribroperidinium asarotum, Site 765, B:417-418

Cribroperidinium leedervillense, Site 765, B:417

Cribroperidinium sp., Site 765, B:417

Crucella cachensis, B:319

Site 765, B:333

Crucella espartoensis, B:319

Site $765, \mathrm{~B}: 333$

Crucella spp., B:319

Site 765, B:333

Crucibiscutum salebrosum, B:360

bipolar distribution, B:42, 356-357, 743

localities found, B:356

morphology, B:40

Site 261, B:359, 378, 741

Site 765, B:36, 350, 356, 368, 741

Site 766, B:350, 356, 741

Cruciellipsis cuvillieri, B:767

Site 261, B:40, 359, 370, 378, 741

Site $765, \mathrm{~B}: 36,350-351,367,369,378,730$, 741,743

Site 766, A:299; B:350-351, 359, 741, 743

Cruciellipsis cuvillieri Zone, Site 766, B:450

cruciferum, Histiastrum, B:321

Site 765, B:336

Cruciplacolithus primus Subzone, Site 766, A:291; B:398, 731

Cruciplacolithus tenuis, Site 765, B:727

Cruciplacolithus tenuis Subzone

Site 765, B:727

Site 766, A:291; B:398-399

Crybelosporites striatus Zone, Site 765, A:125

Cryptamphorella macropora, B:319

Site $765, \mathrm{~B}: 333$

Cryptamphorella sp., B:312, 319

Site 765, B:333, 730

cuneata, Ellipsoidella, Site 766, B:278, 289, 294

Cuneus ludbrookae, Site 766, B:289, 293

Cuneus sp., Site 766, B:278

cuvillieri, Cruciellipsis, B:767

Site 261, B:40, 359, 370, 378, 741

Site 765, B:36, 350-351, 367, 369, 378, 730, 741,743

Site 766, A:299; B:350-351, 359, 741, 743

Cyclagelosphaera argoensis n. sp., B:371

Site 261, B:40, 378, 741

Site 765, B:741

Cyclagelosphaera deflandrei, Site 765, B:344345,367

Cyclastrum infundibuliforme, B:319

Site 765, B:334

Cyclastrum sp., B:319

Site $765, \mathrm{~B}: 334$
Cyclicargolithus floridanus Subzone, Site 765, A:123; B:394-395, 725

cylindroides, Laevidentalina, Site 766, B:288

Cyrtocapsa grutterinki, B:320

Cyrtocapsa pseudacerra, B:320

Site 765, B:313, 330, 334

Cyrtocapsa sp., B:320

Site $765, \mathrm{~B}: 313,330,334$

Dapsilidinium pastielsii, Site 765, B:440

davidii, Diconodinium, Site 765, B:417

deflandrei, Aldorfia, Site 765, B:420

deflandrei, Cyclagelosphaera, Site 765, B:344345,367

dehiscens, Globoquadrina, Site 765, A:118

dehiscens, Sphaeroidinella, B:718

Site 765, A:118

delicatus, Amaurolithus, Site 766, A:289

delrioensis, Hedbergella

Site 765, A:120

Site 766, A:294

delrioensis, Praeglobotruncana, Site 766, B:282, 291, 297

Dendrophrya latissima, Site 765, B:253

Dentoglobigerina altispira, Site 765, A:117

depressa, Hippocrepina, Site 765, A:122; B:249, 254, 257

diamphidia diamphidia, Foremanella, B:321 Site 765, B:336

diamphidia hipposidericus, Foremanella, B:321

Site 765, B:336

diaphorogona, Acaeniotyle, B:317, 332

Dicarinella primitiva Zone, Site 765, B:727

Dicasanella primitiva Zone, Site 765, A:120

Diconodinium davidii, Site 765, B:408, 417

Diconodinium davidii Zone, Site 765, A:125; $\mathrm{B}: 407,730$

Dictyococcites bisectus Zone, Site 765, A:123; B:395, 725

Dictyococcites productus, Site 766, A:289

dictyotum, Scriniodinium, Site 766, A:301

Didymocyrtis avita, Site 765, A:126

diffundens, Glomospira, Site 765, B:253

digitalis, Wanea, Site 765, A:126

dilatata, Hyperammina, Site 765, B:254, 257

Dingodinium cerviculum, Site 765, B:419

Dingodinium jurassicum, Site 765, B:419

diplogrammus, Glaukolithus, Site 766, B:367

Discoaster asymmetricus, Site 766, A:289

Discoaster binodosus Subzone, Site 765, A:123. B:394, 725

Discoaster brouweri Zone, Site 765, A:122; B:723

Discoaster diastypus Zone, Site 766, A:289; B:399, 731

Discoaster druggi Subzone, Site 765, A:123; $\mathrm{B}: 395,725$

Discoaster exilis, Site 765, A:123

Discoaster kugleri Subzone, Site 765, A:123

Discoaster lodoensis Zone

Site 765, B:394

Site 766, A:289; B:400, 731

Discoaster mohleri Zone

Site 765, A:123; B:393, 727

Site 766, A:290; B:399, 731

Discoaster multiradiatus Zone

Site 765, A:123; B:393-394, 725, 727

Site 766, B:399

Discoaster neorectus Subzone, Site 765, A:122123

Discoaster nobilis Zone

Site 765, B:393-394

Site 766, B:399, 731
Discoaster quinqueramus Zone, Site 765, A:122; B:723

Discoaster sublodoensis Zone, Site 766, B:731

Discoasteroides kuepperi Subzone

Site 765, A:123; B:394, 725

Site 766, B:400

Dissiliodinium sp., Site 765, B:418

dohertyi, Planulina, Site 765, A:121

Dollidinium sinuosum, Site 765, B:420

Dorothia praehauteriviana, B:765, 767

Site 261, A:122

Site 534, B:764

Dorothia sp., Site 766, B:293

dorysphaeroides, Sethacapsa, B:325

Site 765, B:341

Druggidium apicopaucicum, B:765

duplex, Reophax, Site 765, B:255, 259

dutemplei, Cibicidoides, Site 765, A:121

Duvalia, B:445

Indo-Pacific Province, B:447

Site 765, A:129

Site 766, A:297

Duvalia lobispinosum Zone

Site 761, B:450

Site $763, \mathrm{~B}: 450$

Duvalia sakalava, B:445

Site 766, B:450, 751

dysculum, Belodinium, Site 765, B:420

echigoense, Operculodinium, Site 765, B:435, 439

echiodes, Triactoma, B:326

Eggerella sp., Site 765, B:263

Egmontodinium torynum, Site 765, B:419

Egmontodinium torynum Zone

Site $763, \mathrm{~B}: 450$

Site $765, \mathrm{~A}: 126$

Eiffellithus turriseiffelii

Site 765, A:124; B:383

Site 766, A:291

Eiffellithus turriseiffelii Zone

Site 761, B:97

Site 765, A:124; B:727

Site 766, B:396, 733

Eiffellithus windii, Site 765, B:36, 369

Ellipsoglandulina sp., Site 766, B:278, 294

Ellipsoidella cuneata, Site 766, B:278, 289, 294

embergeri, Zeugrhabdotus, Site 261, B:379

eodela, Campylosphaera, Site 766, B:731

Epelidosphaeridia colligata, Site 765, B:419

Epistomina caracolla, Site 765, B:264

Epitricysta sp., Site 765, B:420

Eprolithus bettenstaedtii n. sp., B:360 Site 766, B:366, 368

Eprolithus floralis, B:357

Site $765, \mathrm{~A}: 124$

Site 766, A:293; B:359, 365-366, 368

Eprolithus sp., Site 766, B:365, 368

erectus, Zeugrhabdotus

Site 261, B:379

Site $765, \mathrm{~A}: 125$

Ericsonia formosa Subzone, Site 765, A:123; B:394, 725

Escharisphaeridia sp., Site 765, B:419

espartoensis, Crucella, B:319

Site $765, \mathrm{~B}: 333$

Eucyrtis columbaria, B:320

Site 765, A:127; B:335

Site 766, A:295

Eucyrtis hanni, B:320

Site $765, \mathrm{~B}: 335$

Eucyrtis micropora, B:320

Site $765, \mathrm{~B}: 335$

Eucyrtis spp., spindle-shaped forms, A:295 
Eucyrtis tenuis, B:320

Site 765, A:127; B:335

Eurycheilostoma hergottensis, Site 766, B:278, 289, 293

Eusyringium columbaria, Site 766, B:311

Eusyringium sp., B:320-321

Site 765, B:313, 330-331, 335

Site 766, B:311

excavata, Tricarinella, Site 766, B:289

excelsa, Hormosina, Site 765, B:254, 259

exilis, Discoaster, Site 765, A:123

Exochosphaeridium arnace, Site 765, B:418

Exochosphaeridium sp., Site 765, B:418

extremus, Globigerinoides, Site 765, A:117, 119

fasciatum, Kleithriasphaeridium, Site 765, B:418

Fasciculithus spp., extinction, A:290

Fasciculithus tympaniformis Zone, Site 765, B:393, 727

Favusella, Site 766, B:284

feifeli, Paalzowella, Site 765, B:264

ferreolensis, Globigerinelloides, Site 765, A:120

fistulosus, Globigerinoides, Site 765, A:117

Flabellites biforaminis, Site 766, B:359, 364, 368

floralis, Eprolithus, B:357

Site 765, A:124

Site 766, A:293; B:359, 365-366, 368

fohsi, Globorotalia, Site 765, A:119

Foremanella diamphidia diamphidia, B:321

Site 765, B:336

Foremanella diamphidia hipposidericus, B:321

Site $765, \mathrm{~B}: 336$

Foremanina sp., Site 765, A:126-127; B:308

fossacincta, Watznaueria, Site 261, B:370

fusca, Psammosphaera, Site 765, B:255, 257

galeata, Aprobolocysta, Site 765, B:420

gandolfii, Spiroplectinella, Site 766, B:273, 287, 292

Gaudryina richteri, Site 766, B:273, 287, 292

Gaudryina sp., Site 765, B:253, 262

gaultensis, Seribiscutum, n. sp., B:360

Site 766, B:363, 368

gaultina, Glomospirella, Site 765, B:253, 258

gaultina, Tritaxia, Site 766, B:273, 287, 292

Gavelinella andersoni, Site 766, B:279, 290, 295

Gavelinella barremiana, Site 766, A:295

Gavelinella indica, Site 766, B:279-280, 290, 295

Gavelinella sp.

Site $765, \mathrm{~B}: 264$

Site 766, B:279-280, 290, 295

Gephyrocapsa oceanica Zone, Site 765, A:122

gladiatoris, Belemnopsis, Ethiopian-Antarctic Subprovince, B:446

Glaukolithus diplogrammus, Site 766, B:367

Globigerina angulisuturalis, Site 765, A:119

Globigerina apertura, Site 765, A:117

Globigerina nepenthes, Site 765, A:117-119

Globigerinelloides aptiense, Site 766, B:280, 290, 295-296

Globigerinelloides bentonensis, Site 766, B:280, 290, 296

Globigerinelloides ferreolensis, Site 765, A:120

Globigerinelloides sp., Site 766, A:294; B:280

Globigerinoides extremus, Site 765, A:117, 119

Globigerinoides fistulosus, Site 765, A:117

Globigerinoides primordium, Site 765, A:119

Globigerinoides subquadratus, Site 765, A:119

Globigerinoides triloba, Site 765, A:119

Globoquadrina binaiensis, Site 765, A:119

Globoquadrina dehiscens, Site 765, A:118

Globoquadrina venezuelana, Site 765, A:118

Globorotalia cibaoensis, Site 765, A:119
Globorotalia crassaformis, Site 765, A:118

Globorotalia fohsi, Site 765, A:119

Globorotalia inflata

Site 765, A:117

Site 766, A:293

Globorotalia juanai, Site 765, A:119

Globorotalia kugleri, Site 765, A:119

Globorotalia lenguaensis, Site 765, A:119

Globorotalia margaritae, Site 765, A:117-118

Globorotalia mayeri, Site 765, A:119

Globorotalia merotumida, Site 765, A:119

Globorotalia paralenguaensis, Site 765, A:119

Globorotalia peripheroacuta, Site 765, A:119

Globorotalia peripheroronda, Site 765, A:119

Globorotalia plesiotumida, Site 765, A:118-119

Globorotalia praemenardii, Site 765, A:119

Globorotalia puncticulata, Site 766, B:731

Globorotalia tosaensis, Site 765, A:117

Globorotalia truncatulinoides, Site 765, B:723

Globorotalites, Australian distribution, B:284

Globorotalites aptiensis, Site 766, B:290, 294

globosus, Gyroidinoides, Site 766, A:294

Globotruncana aegyptiaca Zone, Site 765, A:120

Globotruncana calcarata Zone, Site 765, A:120

Globotruncana ventricosa, Site 765, A:120

Globotruncanella havanensis

Site 765, A:120

Site 766, A:294

Globotruncanella petaloidea, Site 765, A:120

Globulina prisca, Site 766, B:289

glomerosa, Praeorbulina, Site 765, A:119

Glomospira charoides

Site 261, B:56

Site $765, \mathrm{~B}: 253$

Glomospira diffundens, Site 765, B:253

Glomospira gordialis, Site 765, B:253, 258

Glomospira irregularis, Site 765, B:253, 258

Glomospira serpens, Site 765, B:253, 258

Glomospira sp., Site 765, B:258

Glomospirella gaultina, Site 765, B:253, 258

Glomospirella $\mathrm{sp}$

Site 765, B:253, 258

Site 766, B:287, 292

Gonyaulacysta helicoidea, Site 765, B:418

gordialis, Glomospira, Site 765, B:253, 258

gracilis, Laevidentalina, Site 766, B:288

gracillima, Serovaina, Site 766, B:289, 294

Grantarhabdus coronoadtventis, Site 766, B:365

granulatum, Carpodinium, Site 765, B:417

grutterinki, Cyrtocapsa, B:320

grzybowskii, Kalamopsis, Site 765, B:254, 259

guttata, Lenticulina, Site 765, B:264

Gyroidinoides globosus, Site 766, A:294

Halesium sexangulum, Site 765, A:127; B:308

hamulus, Hemirobulina, Site 766, B:288

hanni, Eucyrtis, B:320

Site $765, \mathrm{~B}: 335$

Haplophragmium inconstans, B:765

Site $765, A: 122$

Haplophragmium lueckei, A;121

Haplophragmoides gigantea Zone, Site 765 , A:121

Haplophragmoides kirki, Site 765, B:253, 260

Haplophragmoides sp., Site 765, B:253-254, 260

Hapsocysta perdictya, Site 765, B:418

Haquis, B:372

havanensis, Cibicidoides, Site 765, A:121

havanensis, Globotruncanella

Site 765, A:120

Site 766, A:294

Hayesites albiensis

Site 765, A:124
Site 766, A:293

Hayesites irregularis, Site 766, B:733

Hedbergella aptica, Site 766, B:281, 291, 296

Hedbergella assemblages, Site 766, B:282-283, 733

Hedbergella delrioensis

Site 370, B:765

Site $765, \mathrm{~A}: 120$

Site 766, A:294

Hedbergella planispira

evolution, B:281-282

Site 765, A:120

Site 766, B:291, 296

Hedbergella punctata, Site 766, A:294; B:282, 291, 297

Hedbergella spp., Site 766, A:294

Hedbergella trocoidea, Site 766, A:294; B:282, 291, 297

Hedbergella yezoana, Site 766, B:291, 297

helenae, Alievium, B:317-318

Site 766, A:295

helicoidea, Gonyaulacysta, Site 765, B:418

Helicopontosphaera selli Zone, Site 765, A:122

Heliolithus kleinpelli Zone, Site 765, B:393

helveticus, Reophax, Site 765, B:255, 259

Hemirobulina hamulus, Site 766, B:288

Herendeenia postprojecta, Site 765, B:417

Hergottella sp., Site 766, B:278, 294

hergottensis, Eurycheilostoma, Site 766, B:278, 289, 293

Heterantyx cretosa, Site 766, B:273, 287, 292

Hibolithes, B:445

Indo-Pacific Province, B:446

regional differentiation, $\mathrm{B}: 446$

Site $763, B: 450$

Site 765, A:129

Site 766, A:297

Hippocrepina depressa, Site 765, A:122; B:249, 254,257

Histiastrum cruciferum, B:321

Site 765, B:336

hofkeri, Conorboides, Site 766, A:295

Holocryptocanium barbui, Site 765, A:128, 130, 142; B:43, 310, 730

Holocryptocanium barbui barbui, B:312, 321

Site 765, B:336

Holocryptocanium spp., Marin Headlands Terrane, B:744

Hormosina crassa, Site 765, B:254, 259

Hormosina excelsa, Site 765, B:254, 259

Hormosina ovulum, Site 765, A:122; B:259

Hormosina ovulum gigantea, Site 765, A:121; B:727

Hormosina ovulum gigantea Zone, Site 765 , B: 727

Hormosinella sp., Site 765, B:254, 259

humerosa, Neogloboquadrina, Site 765, A:117

humilis, Pseudonodosaria, Site 766, B:288

Hyperammina dilatata, Site 765, B:254, 257

Hyperammina sp., Site 765, B:254, 257

Hyperamminoides sp., Site 765, B:257

Hystrichokolpoma rigaudiae, Site 765, B:432, 441

Hystrichosphaeropsis sp., Site 765, B:418

Impagidinium paradoxum, Site 765, B:438

Impagidinium patulum, Site 765, B:438

Impagidinium phlyctaena, Site 765, B:418

Impagidinium sphaericum, Site 765, B:438

Impagidinium spp., Site 765, B:434

Impagidinium strialatum, Site 765, B:438

imperfecta, Batiacasphaera, Site 765, B:419-420

Impletosphaeridium spp., Site 765, B:435 
inconstans, Bulbobaculites, Site 765, B:249, 252253, 260

inconstans, Haplophragmium, B:765

Site 765, A:122

indica, Gavelinella, Site 766, B:279-280, 290, 295

inequiornatum, Prolixosphaeridium, Site 765, B:419

inflata, Clinapertina, Site 766, A:294

inflata, Globorotalia

Site 765, A:117

Site 766, A:293

inflexum, Stephanastrum, B:326

Site 765, B:342

infracretacea, Serovaina, Site 766, B:279, 289, 294

infundibuliforme, Cyclastrum, B:319

Site 765, B:334

intermedia, Berthelina, Site 766, B:279, 290, 294

intermedia, Canninginopsis, Site 765, A:125; B:407, 419

irregularis, Glomospira, Site 765, B:253, 258

irregularis, Hayesites, Site 766, B:733

irregularis, Rucinolithus, Site 766, B:359, 365, 368

israelianum, Operculodinium, Site 765, B:439

Isthmolithus recurvus Subzone, Site 765, B:394, 725

janduchenei, Operculodinium, Site 765, B:430$431,435,439$

jarvisi, Neoflabellina, Site 766, A:294

jeletzkyi, Arenoturispirillina, Site 765, B:252, 258

jonkeri, Belemnopsis, B:444-445, 450

juanai, Globorotalia, Site 765, A:119

jurassicum, Dingodinium, Site 765, B:419

Kaiwaradinium scrutillinum, Site 765, A:126; B:416

Kaiwaradinium scrutillinum Zone, Site 765 , A: 126

Kalamopsis grzybowskii, Site 765, B:254, 259

Kalamopsis sp., Site 765, B:259

Kallosphaeridium sp., Site 765, B:419

kennedyi, Corollithion, Site 765, A:124; B:727

kirki, Haplophragmoides, Site 765, B:253, 260

Kleithriasphaeridium fasciatum, Site 765, B:418

kugleri, Globorotalia, Site 765, A:119

laboriosus, Tripocalpis, B:326

Site 765, B:342

lacunosa, Pseudoemiliania, Site 765, A:122

Laevidentalina cylindroides, Site 766, B:288

Laevidentalina gracilis, Site 766, B:288

Laevidentalina nana, Site 766, B:288

Laevidentalina oligostegia, Site 766, B:288

laevigata, Psammosphaera, Site 765, B:257

laffittei, Rotelapillus, Site 766, B:366

Lagenammina sp., Site 765, B:254, 257

latissima, Dendrophrya, Site 765, B:253

leedervillense, Cribroperidinium, Site 765, B:417

leiostraca, Sethocapsa, B:325

Site 765, A:127; B:308, 341

lemniscata, Nematosphaeropsis, Site 765, B:441

lenguaensis, Globorotalia, Site 765, A:119

Lenticulina barremiana, Site 766, B:764

Lenticulina guttata, Site 765, B:264

Lenticulina nodosa

Site 370, B:764

Site 765, B:264

Lenticulina saxonica, Site 765, B:264

Lenticulina subalata, Site 765, B:264

Lenticulina subangulata, A:122
Leptodinium sp., Site 765, B:418, 420

lilyae, Pseudodictyomitra, B:324

linearis, Rhabdammina, Site 765, B:257

Lingulina buddencanyonensis, Site 766, B:288

Lingulina loryi, Site 765, B:264

Lingulina sp., Site 766, B:293

Lingulodinium machaerophorum, Site. 765, B:441

Lingulogavelinella

Australian distribution, B:284

Site 766, A:295

Lingulonodosaria loryi, Site 766, B:288

Lingulonodosaria nodosaria, Site 766, B:288

Lingulonodosaria sp., Site 766, B:288, 293

Lithatractus pusillus, B:321

Lithraphidites bollii Zone, Site 765, A:124

Lithraphidites carniolensis

Site 261, B:351

Site $765, \mathrm{~B}: 350,730$

litterarius, Chiastozygus, B:767

Site 766, B:364, 368

Lituotubo spp., Site 765, B:254

longa, Pyrulina, Site 765, B:264

longispinigerum, Operculodinium, Site 765, B:431, 435, 439

loryi, Lingulina, Site 765, B:264

loryi, Lingulonodosaria, Site 766, B:288

Lotharingius contractus Subzone, Site 765, A:125

Lotharingius velatus, Site 765, A:125

Lucianorhabdas maleformis Zone

Site 765, A:124; B:383

Site 766, A:291; B:396, 733

ludbrookae, Cuneus, Site 766, B:289, 293

lueckei, Haplophragmium, A:121

machaerophorum, Lingulodinium, Site 765, B:441

macropora, Cryptamphorella, B:319

Site 765, B:333

macwhaei, Muderongia, Site 765, B:407

magna, Cassiculosphaeridia, Site 765, B:416

malleola, Parvicingula, B:322

Site 765, B:337

Site 766, A:295

manivitae, Watznaueria, B:371-372

Site 261, B:25, 40, 56, 370, 378, 741

Site 765, A:124, 246; B:36, 42, 369, 378, 741

Manivitella pemmatoidea, Site 766, B:364

margaritae, Globorotalia, Site 765, A:117-118

Marginotruncana sigali Zone, Site 765, A:120

Marginulopsis collignoni

Site 416, A:295

Site 766, A:295; B:735

Marssonella Association

Australia, B:283

biogeography, B:283

global distribution, B:284

Marssonella oxycona, Site 766, B:288, 293

Marssonella sp., Site 765, B:254, 263

Marthasterites furcatus Zone

Site 765, A:124; B:383

Site 766, A:291; B:396, 733

matalosa, Vagalapilla, B:360

Indo-Pacific Region, B:351, 356

Site 261, B:40

Site 765, A:124; B:36, 350, 369, 378, 743

Site 766, B:368, 743

mayeri, Globorotalia, Site 765, A:119

mckannai, Acarinina, Site 766, A:293

media, Angulobracchia, B:318

Site 765, B:332

mediocrix, Amphipyndas, B:318

Meiourogonyaulax sp., Site 765, B:416, 419-420

Meiourogonyaulax stoveri, Site 765, B:419
Melitasphaeridium choanophorum, Site 765 , B:431, 440

membranaceus, Spiniferites, Site 765, B:438

merotumida, Globorotalia, Site 765, A:119

mexicana, Conusphaera, Site 261, B:370

mexicana, Planulina, Site 765, A:121

mexicana minor, Conusphaera

Site 261, B:37, 40, 741

Site $765, \mathrm{~B}: 741$

Micrantholithus hoschulzii Zone, Site 765, A:124

micropapillata, Batiacasphaera, Site 765, B:440

micropodum, Batioladinium, Site 765, B:418

micropora, Eucyrtis, B:320

Site 765, B:335

Microrhabdulus decoratus Zone

Site 761, B:97

Site 765, A:124; B:727

Site 766, A:291; B:733

Microstaurus chiastius, Site 765, B:344-345

Microstaurus quadratus, Site 765, B:344-345

Micula decussata Zone

Site 765, A:124; B:727

Site 766, A:291; B:733

milamensis, Rugoglobigerina, Site 766, A:294

minutus, Reophax, Site 766, A:295

mirabilis, Spiniferites, Site 765, B:438

Mirifusus chenodes, B:321

Site $765, \mathrm{~B}: 336$

moluccana, Belemnopsis, B:445

Site $763, \mathrm{~B}: 450$

Morozovella subbotinae complex, Site 766, A:293

Morozovella velascoensis, Site 766, A:293

Muderongia australis, Site 765, B:416

Muderongia australis Zone

Argo Abyssal Plain, B:42

Site 765, A:125; B:408, 410, 730

Site 766, A:297; B:733, 735

Muderongia macwhaei, Site 765, B:407

Muderongia testudinaria Zone, Site 766, B:735

Muderongia tetracantha, Site 765, B:416

Muderongia tetracantha Zone, Site 765, A:125; B:730

multiloculata, Ticinella, Site 257, B:284

Multispinula quantra, Site 765, B:441

nana, Laevidentalina, Site 766, B:288

nannina, Praebulimina, Site 766, B:289, 293

Nannoconus, B:754-755

biogeographic differentiation, B:741, 743

exclusion from Argo Abyssal Plain, B:42

Napora spp., B:321

Nematosphaeropsis lemniscata, Site 765, B:441

Nematosphaeropsis spp., Site 765, B:434

Nematosphaeropsis wrennii n. sp., Site 765 , B: 435,440

neocomica, Phoberocysta, B:765, 767

Site 765, B:408

neocomiensis, Verneuilinoides, Site 765, A:122; B:249, 256, 263

Neoflabellina jarvisi, Site 766, A:294

Neogloboquadrina acostaensis, coiling change, A:119

Neogloboquadrina humerosa, Site 765, A:117

nepenthes, Globigerina, Site 765, A:117-119

nephroides, Selenopemphix, Site 765, B:441

Nephrolithus frequens Zone, Site 766, A:291; B:398, 731

Nephrolithus frequens Zone/ Cruciplacolithus primus Subzone, Site 766, B:398

neptuni, Achomosphaera, Site 765, B:418

neyrivensis, Produvalia, Site 765, A:129

nitida, Candeina, Site 765, A:119

nodosa, Lenticulina 
Site 370, B:764

Site 765, B:264

nodosaria, Lingulonodosaria, Site 766, B:288

Nodosaria obscura, Site 765, B:264

Nothofagus, Australia SE, B:423

Nuttallides umbonifera, Site 766, A:294

Obesacapsula, B:321

Obesacapsula rotunda, Site 765, A:128; B:730

Obesacapsula verbana, B:322

Site 765, B:310, 336

obscura, Nodosaria, Site 765, B:264

obscura, Pyramidulina, Site 766, B:288

Occisucysta tenuiceras, Site 765, B:417

Odontochitina operculata, B:765

Site 765, B:419

Odontochitina operculata Zone

Argo Abyssal Plain, B:42

Site 765, A:125; B:407-408, 410, 730

Oligosphaeridium asterigerum, Site 765, B:416

Oligosphaeridium poculum, Site 765, B:419

oligostegia, Laevidentalina, Site 766, B:288

operculata, Odontochitina, B:765

Site 765, B:419

Operculodinium echigoense, Site 765, B:435, 439

Operculodinium israelianum, Site 765, B:439

Operculodinium janduchenei, Site 765, B:430$431,435,439$

Operculodinium longispinigerum, Site 765, B: $431,435,439$

orbiculare, Stephanastrum, B:326

Site 765, B:342

Orbiculiforma railensis, B:322

Site 765, B:336

Orbiculiforma spp., B:322

Site 765, B:336

orca, Sethocapsa, B:325

Site $765, \mathrm{~B}: 341$

Osangularia schloenbachi, Site 766, B:279, 290, 294

ovulum, Hormosina, Site 765, A:122; B:259

ovulum gigantea, Hormosina, Site 765, A:121; B:727

oxycona, Marssonella, Site 766, B:288, 293

Oxyteuthis, morphological trends, B:446

Paalzowella feifeli, Site 765, B:264

palmula, Systematophora, Site 765, B:416

Pantanellium squinaboli cantuchapai, B:322 Site 765, B:337

Pantanellium squinaboli squinaboli, B:322

Site 765, B:337

paradoxum, Impagidinium, Site 765, B:438

paralenguaensis, Globorotalia, Site 765, A:119

Paratrochammina sp., Site 765, B:254, 262

Paratrochamminoides sp., Site 765, B:254, 261

parcus constrictus, Aspidolithus

Site 765, A:123; B:727

Site 766, A:291; B:732

parcus parcus, Aspidolithus, Site 766, A:291

Paronaella sp., B:322

Site 765, B:331, 337

parri, Plectofrondicularia, Site 765, A:121

Parvicingula malleola, B:322

Site $765, \mathrm{~B}: 337$

Site 766, A:295

Parvicingula sp., B:313, 322-323, 744

Site 765, B:330, 338

Parvicingula sphaerica, B:322

parvidentatum, Repagulum, Site 765, B:36, 369

parvispinum, Prolixosphaeridium, Site 765, B:419

parvissimus, Bolivinoides, Site 765, B:727

pastielsii, Dapsilidinium, Site 765, B:440
Patellula sp., B:323

Site 765, B:339

patulum, Impagidinium, Site 765, B:438

pemmatoidea, Manivitella, Site 766, B:364

Pentadinium sp., Site 765, B:440

perdictya, Hapsocysta, Site 765, B:418

Peregrinocceras albidum ammonite Zone, Site $765, \mathrm{~B}: 350$

peripheroacuta, Globorotalia, Site 765, A:119

peripheroronda, Globorotalia, Site 765, A:119

perspicua, Stichocapsa, B:326

Site 765, B:342

petaloidea, Globotruncanella, Site 765, A:120

Petasiforma sp., B:323-324

phlyctaena, Impagidinium, Site 765, B:418

Phoberocysta burgeri Zone, Site 766, A:297; B:735

Phoberocysta neocomica, B:765, 767

Site 765, B:408

placacantha, Systematophora, Site 765, A:125; B: 432

planispira, Hedbergella

evolution, B:281-282

Site 765, A:120

Site 766, B:291, 296

Planolites, Site 261, B:27

Planomalina buxtorfi

Site 765, A: 120

Site 766, A:294; B:280-281, 290, 296

Planomalina buxtorfi Zone, Site 766, A:294

Planomalina praebuxtorfi, Site 766, B:280, 290, 296

Planomalina praebuxtorfi Zone, Site 766, B:282, 733

Planularia bradyana, Site 766, B:289

Planulina dohertyi, Site 765, A:121

Planulina mexicana, Site 765, A:121

Planulina renzi, Site 765, A:121

Plectofrondicularia parri, Site 765, A:121

Plectofrondicularia vaughani, Site 765, A:121

Plectorecurvoides alternans Zone, Site 765 ,

$$
\text { A:121 }
$$

plesiotumida, Globorotalia, Site 765, A:118-119

Pleurostomella reussi, Site 766, B:278, 289, 294

poculum, Oligosphaeridium, Site 765, B:419

Podocapsa amphitreptera, B:324

Site 765, A:128, 141-142; B:308-309, 339

Podocapsa sp., B:324

Site 765, B:339

Polysphaeridium zoharyi, Site 765, B:429-430, 435, 441

postprojecta, Herendeenia, Site 765, B:417

Praebulimina nannina, Site 766, B:289, 293

praebuxtorfi, Planomalina, Site 766, B:280, 290, 296

Praeconocaryomma prisca, B:324

Site 765, B:339

Site 766, A:296-297; B:311

Praeconocaryomma sp., B:324

Site 765, B:331, 339

Praecystammina sp., Site 765, B:254, 261

Praediscosphaera columnata Zone, Site 766 , B:733

Praedorothia praehauteriviana, Site 765, B:249$250,255,262$

Praedorothia praehauteriviana Assemblage, Site $765, \mathrm{~B}: 245,249$

Praedorothia sp., Site 765, B:255, 262

Praeglobotruncana delrioensis, Site 766, B:282, 291, 297

praehauteriviana, Dorothia, B:765, 767

Site 261, A:122

Site 534, B:764
| praehauteriviana, Praedorothia, Site 765, B:249250, 255, 262

praemenardii, Globorotalia, Site 765, A:119

Praeorbulina glomerosa, Site 765, A:119

Praeoxyteuthis, morphological trends, B:446

Prediscosphaera columnata

Site 765, B:359

Site 766, A:293; B:359

Prediscosphaera columnata Zone

Site 765, A:124; B:383, 727

Site 766, B:396

Prediscosphaera spinosa, Site 766, B:368

primalis, Pulleniatina, Site 765, A:119

primitivum, Seribiscutum, B:357-358

distribution, B:358, 743

Site 765, B:741, 743, 754

Site 766, A:342; B:368, 741, 743, 754

primordium, Globigerinoides, Site 765, A:119

prisca, Globulina, Site 766, B:289

prisca, Praeconocaryomma, B:324

Site 765, B:339

Site 766, A:296-297; B:311

prismatium, Pterocanium, reworking, A:126

productus, Dictyococcites, Site 766, A:289

Produvalia neyrivensis, Site 765, A:129

Produvalia sp., Site 765, A:129

Prolixosphaeridium inequiornatum, Site 765 , B:419

Prolixosphaeridium parvispinum, Site 765, B:419

protindica, Scheibnerova, Site 766, B:280, 290, 295

Psammosphaera fusca, Site 765, B:255, 257

Psammosphaera laevigata, Site 765, B:257

Psammosphaera sp., Site 765, B:255

pseudacerra, Cyrtocapsa, B:320

Site 765, B:313, 330, 334

Pseudobolivina sp., Site 765, B:255, 263

Pseudoceratium turneri, Site 765, B:416

Pseudodictyomitra lilyae, B:324

Pseudoemiliania lacunosa, Site 765, A:122

Pseudoeucyrtis sp., B:324

Site 765, B:330, 340

Pseudogaudryinella sp., Site 766, B:273, 287,292

Pseudohastigerina barbadoensis, Site 765, A:119

Pseudonodosaria humilis, Site 766, B:238

Pseudoreophax cisownicensis, Site 765, B:255, 263

Pseudoreophax sp., Site 765, B:249

Pseudoreophax-Thalmannammina-Haplophragmoides kirki Assemblage, Site 765, B:242-243, 245

Pseudothalmanninella ticinensis-Planomalina buxtorfi Zone, Site 766, B:282, 733

Psilocitharella recta, Site 766, B:289

Pterocanium prismatium, Site 765, A:126

ptomatis, Senoniasphaera, Site 765, B:419

Pulleniatina primalis, Site 765, A:119

Pulleniatina spp., Site 765, A:118

punctata, Hedbergella, Site 766, A:294; B:282, 291, 297

puncticulata, Globorotalia, Site 766, B:731

pusillus, Lithatractus, B:321

Pyramidulina obscura, Site 766, B:288

Pyramidulina sceptrum, Site 766, B:288

Pyramidulina sp., Site 766, B:288

Pyrulina longa, Site 765, B:264

Pyxidinopsis sp., Site 765, B:440

quadratus, Microstaurus, Site 765, B:344-345

Quadrimorphina, Site 766, A:294

Quadrimorphina allomorphinoides, Site 766, B:290, 294

Quadrum gartneri Zone 
Site 765, A:124-125; B:383, 727

Site 766, A:291; B:396, 733

Quadrum nitidum Zone, Site 766, B:397

Quadrum trifidum Zone

Site 765, A:123-124; B:392, 727

Site 766, A:291; B:397-398, 732

quantra, Multispinula, Site 765, B:441

quinqueloba, Trochammina, B:765, 767

Site 765, A:122; B:249-250, 256, 262

railensis, Orbiculiforma, B:322

Site 765, B:336

ramosus, Spiniferites, Site 765, B:438

Ramulina aculeata, Site 765, B:264

recta, Psilocitharella, Site 766, B:289

reidii, Stelladinium, Site 765, B:441

Reinhardtites anthophorus Zone

Site 765, A:124; B:727

Site 766, A:291; B:397, 732-733

Reinhardtites anthophorus-Lucianorhabdus cayeuxii zones, Site 766, B:397

Remesella sp.

intrachamber partitions, B:273

Site $766, \mathrm{~B}: 287$

renzi, Planulina, Site 765, A:121

Reophax duplex, Site 765, B:255, 259

Reophax helveticus, Site 765, B:255, 259

Reophax minutus, Site 766, A:295

Reophax sp., Site 765, B:245, 255, 259

Repagulum parvidentatum, Site 765, B:36, 369

Repmania corona, Site 766, B:287, 292

reticulatum, Batioladinium, Site 765, B:418

Reticulofenestra hillae Subzone, Site 765, A:123; B: 725

Reticulofenestra pseudoumbilica Zone, Site 765 , A:122; B:723

reticulosa, Vaginulinopsis, Site 765, B:264

Reussella szajnochae, Site 766, A:294

reussi, Pleurostomella, Site 766, B:278, 289, 294

Rhabdammina linearis, Site 765, B:257

Rhabdammina sp., Site 765, B:255, 257

Rhagodiscus angustus

Site 765, B:359, 368

Site 766, B:351, 359, 366

Rhagodiscus angustus Zone

Site 765, B:350-351, 730

Site $766, \mathrm{~B}: 351$

Rhagodiscus asper, B:743

Rhizammina sp., Site 765, B:256-257

richteri, Gaudryina, Site 766, B:273, 287, 292

rigaudiae, Hystrichokolpoma, Site 765, B:432, 441

Ristola sp., B:313, 324-325, 340, 744

Rotalipora appenninica, Site 766, B:282, 291, 297

Rotalipora appenninica Zone, Site 766, B:282

Rotalipora cushmani Zone

Site 765, A:120

Site 766, A:294; B:733

Rotalipora reicheli Zone, Site 765, A:120

Rotalipora ticinensis, Site 766, B:282, 291, 297

Rotelapillus crenulatus, Site 766, B:366

Rotelapillus laffittei, Site 766, B:366

rotunda, Obesacapsula, Site 765, A:128; B:730

Rucinolithus irregularis, Site 766, B:359, 365, 368

Rucinolithus wisei, B:767

Site $765, \mathrm{~B}: 350$

Rugoglobigerina milamensis, Site 766, A:294

Saccammina sp., Site 765, B:256-257

Saccocoma, Alpine-Mediterranean Tethyan Realm B:751

sakalava, Duvalia, B:445
Site $766, \mathrm{~B} \cdot 450$

salebrosum, Crucibiscutum, B:360

distribution, B:42, 356-357, 743

morphology, B:40

Site 261, B:359, 378,741

Site 765, B:36, 350, 356, 368, 741

Site 766, B:350, 356,741

salebrosum, Seribiscutum, B:754

Saracenaria spinosa, Site 766, B:289

saxonica, Lenticulina, Site 765, B:264

Scampanella cornuta, Site 766, B:367

sceptrum, Pyramidulina, Site 766, B:288

Schackoina cenomana, Site 766, B:290, 296

Scheibnerova, Australian distribution, B:284

Scheibnerova protindica, Site 766, B:280, 290, 295

schloenbachi, Osangularia, Site 766, B:279, 290,

scitula, Astacolus, Site 766, B:278, 288, 293

Scriniodinium attadalense, Site 765, B:417

Scriniodinium dictyotum, Site 766, A:301

scrutillinum, Kaiwaradinium, Site 765, A: 126; B:416

scutum, Yalkalpodinum, Site 765, B:416

Selenopemphix nephroides, Site 765, B:441

selmensis, Tappanina, Site 766, A:294

seminulina, Sphaeroidinellopis, Site 765, A:117

semireticulata, Aragonia, Site 766, A:294

Senoniasphaera ptomatis, Site 765, B:419

Senoniasphaera tabulata, Site 766, A:297

Senoniasphaera tabulata Zone, Site 766, B:735

septemporatus, Cecrops, B:319

Seribiscutum gaultensis n. sp., B:360

Site 766, B:363, 368

Seribiscutum primitivum, B:357-358

distribution, B:358, 743

Site $765, \mathrm{~B}: 741,743,754$

Site 766, A:342; B:368, 741, 743, 754

Seribiscutum salebrosum, B:754

Serovaina gracillima, Site 766, B:289, 294

Serovaina infracretacea, Site 766, B:279, 289, 294

Serovaina sp., Site 766, B:278

serpens, Glomospira, Site 765, B:253, 258

Sethocapsa cetia, B:325

Site 765, A:128, 141-142; B:43, 341, 730

Sethocapsa dorysphaeroides, B:325

Site 765, B:341

Sethocapsa euganea Zone

Site 765, A:127; B:308, 730

Site 766, A:295; B:311, 733

Sethocapsa leiostraca, B:325

Site 765, A:127; B:308, 341

Sethocapsa orca, B:325

Site 765, B:341

Sethocapsa sp., B:325

Site $765, \mathrm{~B}: 341$

Sethocapsa trachyostraca trachyostraca, B:325

sexangulum, Halesium, Site 765, A:127; B:308

signata, Broinsonia, Site 766, B:364

silvaradion, Corollithion, Site 511, B:356

simplex, Broomea, Site 765, B:418

simplicissima, Clavihedbergella, Site 766, B:282, 290, 296

sinuosum, Dollidinium, Site 765, B:420

Siphonina tenuicarinata, Site 765, A:121

slitera, Archaeodictyomitra, Site 765, B:730

Speetonia colligato

Site 261, B:741

Site $765, \mathrm{~B}: 350,367,730,741,743$

Site $766, \mathrm{~B}: 741,743$

sphaerica, Parvicingula, B:322

sphaericum, Impagidinium, Site 765, B:438
Sphaeroidinella dehiscens, B:718

Site 765, A:118

Sphaeroidinellopsis seminulina, Site 765, A:117

Sphenolithus heteromorphus Zone, Site 765 . A: $123 ; \mathrm{B}: 725$

Spinidinium styloniferum, Site 766, A:297

Spiniferites

Site 765, B:407

spore/pollen ratio and, B:408, 410

Spiniferites bulloideus, Site 765, B:429-430

Spiniferites membranaceus, Site 765, B:438

Spiniferites mirabilis, Site 765, B:438

Spiniferites ramosus, Site 765, B:438

Spiniferites sp., Site 765, B:420

spinosa, Prediscosphaera, Site 766, B:368

spinosa, Saracenaria, Site 766, B:289

Spiroplectammirus cretosa, Site 766, B:273

Spiroplectammina sp., Site 765, B:256

Spiroplectinella gandolfii, Site 766, B:273, 287, 292

Spongaster tetras tetras, Site 766, A:295

Spongocapsula sp., B:325-326

Site 765, B:341

Spongotripus trigonus, B:326

Site 765, B:341

squamata clavata, Trochammina, Site 765, B:252

squinaboli cantuchapai, Pantanellium, B:322

Site 765, B:337

squinaboli squinaboli, Pantanellium, B:322

Site $765, \mathrm{~B}: 337$

Stelladinium reidii, Site 765 , B:441

Stephanastrum inflexum, B:326

Site 765, B:342

Stephanastrum orbiculare, B:326

Site 765, B:342

Stephanolithion bigotii, Site 261, A:7; B:37, 40, 42, 351, 378

Stephanolithion bigotii bigotii, Site 261, B:369370

Stephanolithion bigotii Zone, Site 765, A:125

Stephanolithion bigotii-Conusphaera mexicana minor Assemblage, Site 261, B:527

Stichocapsa perspicua, B:326

Site 765, B:342

Stoliczkaia disparammonoid Zone, Site 766 , B:282

stoveri, Meiourogonyaulax, Site 765, B:419

stradneri, Vekshinella, Site 766, B:363-364

strialatum, Impagidinium, Site 765, B:438

striatum, Tegumentum

Site 261, B:40, 370

Site $765, \mathrm{~B}: 350$

Site $766, B \cdot 350-351,359,368$

styloniferum, Spinidinium, Site 766, A:297

subalata, Lenticulina, Site 765, B:264

subangulata, Lenticulina, A:122

subquadratus, Globigerinoides, Site 765, A:119

Subreophax sp., Site 765, B:256, 259

Systematophora arealata, Site 765, B:416

Systematophora palmula, Site 765, B:416

Systematophora placacantha, Site 765, A:125; $\mathrm{B}: 432$

szajnochae, Reussella, Site 766, A:294

tabulata, Senoniasphaera, Site 766, A:297

Tanyosphaeridium sp., Site 765, B:419

Tappanina selmensis, Site 766, A:294

Tappanina sp.

morphology, B:278

Site 766, B:293

Tegumentum spp., Site 766, A:293, B:735

Tegumentum striatum

Site 261, B:40, 370 
Site 765, B:350, 359

Site 766, B:350-351, 368

Tegumentum tripes, Site 765, B:730

tenuicarinata, Siphonina, Site 765, A:121 tenuiceras, Occisucysta, Site 765, B:417

tenuis, Cruciplacolithus, Site 765, B:727 tenuis, Eucyrtis, B:320

Site 765, A:127; B:335

tenuissimus, Ammodiscus, Site 765, B:252, 258

tetracantha, Muderongia, Site 765, B:416

tetras tetras, Spongaster, Site 766, A:295

Thalmannammina sp., Site 765, B:256, 261

Thalmanninella appenninica-Planomalina buxtorfi Zone, Site 766, B:282, 733

Thanarla conica, Site 765, A:129-130

Ticinella

Australia, B:284

Site 327, B:755

Site 511, B:755

Ticinella multiloculata, Site 257, B:284

ticinensis, Rotalipora, Site 766, B:282, 291, 297

torynum, Egmontodinium, Site 765, B:419

tosaensis, Globorotalia, Site 765, A:117

trachyostraca trachyostraca, Sethocapsa, B:325

Tranolithus phacelosus Zone

Site 765, A:123, 125; B:727

Site 766, A:291; B:398, 732

Triactoma echiodes, B:326

Tribrachiatus contortus Subzone, Site 765, B:393

Tricarinella excavata, Site 766, B:289

Tricolocapsa sp., B:326

Site $765, \mathrm{~B}: 342$

trigonus, Spongotripus, B:326

Site 765, B:341

triloba, Globigerinoides, Site 765, A:119

Trinchodinium castanea, Site 765, B:418

tripes, Tegumentum, Site 765, B:730

Tripocalpis laboriosus, B:326

Site 765, B:342

Tristix acutiangulata

Site 416, A:295

Site 766, A:295; B:735

Tritaxia gaultina, Site 766, B:273, 287, 292

trizonalis, Acanthocircus, B:317

trizonalis dicranacanthos, Acanthocircus, B:317

Trochammina abrupta, Site 765, A:122; B:250, 256, 261-262

Trochammina abrupta-Bulbobaculites inconstans-Ammobaculoides Assemblage, Site $765, \mathrm{~B}: 245$

Trochammina quinqueloba, B:765, 767

Site 765, A:122; B:249-250, 256,262

Trochammina quinqueloba Assemblage, Site 765, B: 242

Trochammina sp., Site 765, B:256, 262

Trochammina squamata clavata, Site 765 , B:252

Trochamminoides sp., Site 765, B:256, 261

trocoidea, Hedbergella, Site 766, A:294; B:282, 291, 297 truncatulinoides, Globorotalia, Site 765, B:723

Tuberculodinium vancampoae, Site 765, B:441

Tubodiscus verenae, B:767

Site 261, A:124; B:40, 370, 378, 741, 764

Site 765, A:124; B:36, 369, 730, 741, 743

Site 766, B:741, 743

Tubotuberella vlamingii, Site 765, B:418

turneri, Pseudoceratium, Site 765, B:416

turriseiffelii, Eiffellithus

Site 765, A:124; B:383

Site 766, A:291

Turritellella sp., Site 765, B:256, 258

tutulosum, Adnatosphaeridium, Site 765, B:418

umbilicata, Acaeniotyle, B:317

Site 765, B:332

umbonifera, Nuttallides, Site 766, A:294

Uvigerinammina sp., Site 765, B:256, 263

Vagalapilla matalosa, B:360

Indo-Pacific Region, B:351, 356

Site 261, B:40

Site 765, A:124; B:36, 350, 369, 378, 743

Site 766, B:368, 743

Vaginulina sp., Site 766, B:293

Vaginulinopsis reticulosa, Site 765, B:264

Vaginulinopsis sp., Site 765, B:264

vancampoae, Tuberculodinium, Site 765, B:441

vaughani, Plectofrondicularia, Site 765, A: 121

Vekshinella stradneri, Site 766, B:363-364

velascoensis, Morozovella, Site 766, A:293

velatus, Lotharingius, Site 765, A:125

venezuelana, Globoquadrina, Site 765, A:118

ventricosa, Globotruncana, Site 765, A:120

verbana, Obesacapsula, B:322

Site 765, B:310, 336

verenae, Tubodiscus, B:767

Site 261, A:124; B:40, 370, 378, 741, 764

Site 765, A:124; B:36, 369, 730, 741, 743

Site $766, \mathrm{~B}: 741,743$

Verneuilinidae sp., Site 766, B:273

Verneuilinoides neocomiensis, Site 765, A:122; B:249, 256, 263

Verneuilinoides neocomiensis Zone, Site 765, A: 122

Verneuilinoides sp., Site 765, B:256, 263

Vitorfus brustolensis, B:326

vlamingii, Tubotuberella, Site 765, B:418

Wanaea digitalis, Site 765, A:126

Wanaea sp., Site 765, B:408

Watznaueria barnesae, B:350 Site 261, B:37

Watznaueria britannica

Site 261, B:37

Site $765, \mathrm{~A}: 125$

Site 766, B:365

Watznaueria britannica Zone, Site 765, A:125

Watznaueria fossacincta, Site 261, B:370
Watznaueria manivitae, B:371-372

Site 261, B:25, 40, 56, 370, 378, 741

Site 765, A:124, 246; B:36, 42, 369, 378, 741

Watznaueria sp., B:754

Site 765, B:8

whitei, Cometodinium, Site 765, B:420

windii, Eiffellithus, Site 765, B:36, 369

wisei, Rucinolithus, B:767

Site $765, \mathrm{~B}: 350$

wrennii, Nematosphaeropsis, n. sp., Site 765 , B: 435,440

Xitus sp., B:326

Site $765, \mathrm{~B}: 342,730$

Yalkalpodinum scutum, Site 765, B:416

yezoana, Hedbergella, Site 766, B:291, 297

Zeugrhabdotus cooperi n. sp., B:372-373

Site 261, B:29, 379,741

Site $765, \mathrm{~B}: 741$

Zeugrhabdotus embergeri, Site 261, B:379

Zeugrhabdotus erectus

Site 261, B:379

Site $765, \mathrm{~A}: 125$

Zeugrhabdotus sp., Site 261, B:379

zoharyi, Polysphaeridium, Site 765, B:429-430, 435,441

zones (with letter prefixes)

CP5, B:727

N4, A:119; B:725

N5, A:119, 121; B:725

N8, A:119; B:725

N9, A:119

N10, A:119

N11, A:119

N12, A:119

N13, A:119

N14, A:119

N15, A:119

N16, A:119

N17, A:118-119

N18, A:41, 118; B:718

N19, A:41, 118, 293; B:718

N20, A:41, 118; B:718

N21, A: 117

N22, A:293

P4, A:293

P5, A:120, 293-294

P6, A:120, 293-294; B:731

P7, A:120, 293; B:731

P8, A:120, 294; B:731

P9, A:120

P10, A:120

P18, A:119

P19, A:119

P22, A:119 